\title{
Prolyl oligopeptidase inhibition activates autophagy via protein phosphatase $2 \mathrm{~A}$
}

\section{Svarcbahs, Reinis}

2020-01

Svarcbahs , R, Jäntti , M , Kilpeläinen , T , Julku , U , Urvas , L , Kivioja , S , Norrbacka , S

\& Myöhänen , T 2020 , ' Prolyl oligopeptidase inhibition activates autophagy via protein

phosphatase 2A ', Pharmacological Research, vol. 151, 104558 . https://doi.org/10.1016/j.phrs.2019.104558

http://hdl.handle.net/10138/321756

https://doi.org/10.1016/j.phrs.2019.104558

acceptedVersion

Downloaded from Helda, University of Helsinki institutional repository.

This is an electronic reprint of the original article.

This reprint may differ from the original in pagination and typographic detail.

Please cite the original version. 


\title{
Prolyl oligopeptidase inhibition activates autophagy via protein phosphatase $2 \mathrm{~A}$
}

\author{
Reinis Svarcbahs ${ }^{1}$, Maria Jäntti ${ }^{1}$, Tommi Kilpeläinen, Ulrika H. Julku, Lauri Urvas, Saara Kivioja, \\ Susanna Norrbacka, Timo T. Myöhänen* \\ Division of Pharmacology and Pharmacotherapy/Drug Research Program, Faculty of Pharmacy, University of Helsinki, Finland
}

\section{A R T I C L E I N F O}

Chemical compounds studied in this article: Bafilomycin A1 (PubChem CID: 6436223) FTY-720 (fingolimod, PubChem CID: 107970) KYP-2047 (PubChem CID: 11198569) Okadaic acid (PubChem CID: 446512) PP242 (PubChem CID: 135565635) Rapamycin (sirolimus, PubChem CID: 5284616)

\section{Keywords:}

Neurodegeneration

Alzheimer's disease

Parkinson's disease

Protein phosphatase 2 phosphatase activator

Protein phosphatase methylesterase 1

Serine protease

\begin{abstract}
A B S T R A C T
Prolyl oligopeptidase (PREP) is a serine protease that has been studied particularly in the context of neurodegenerative diseases for decades but its physiological function has remained unclear. We have previously found that PREP negatively regulates beclin1-mediated macroautophagy (autophagy), and that PREP inhibition by a small-molecule inhibitor induces clearance of protein aggregates in Parkinson's disease models. Since autophagy induction has been suggested as a potential therapy for several diseases, we wanted to further characterize how PREP regulates autophagy. We measured the levels of various kinases and proteins regulating beclin1-autophagy in HEK-293 and SH-SY5Y cell cultures after PREP inhibition, PREP deletion, and PREP overexpression and restoration, and verified the results in vivo by using PREP knock-out and wild-type mouse tissue where PREP was restored or overexpressed, respectively. We found that PREP regulates autophagy by interacting with protein phosphatase 2A (PP2A) and its endogenous inhibitor, protein phosphatase methylesterase 1 (PME1), and activator (protein phosphatase 2 phosphatase activator, PTPA), thus adjusting its activity and the levels of PP2A in the intracellular pool. PREP inhibition and deletion increased PP2A activity, leading to activation of deathassociated protein kinase 1 (DAPK1), beclin1 phosphorylation and induced autophagy while PREP overexpression reduced this. Lowered activity of PP2A is connected to several neurodegenerative disorders and cancers, and PP2A activators would have enormous potential as drug therapy but development of such compounds has been a challenge. The concept of PREP inhibition has been proved safe, and therefore, our study supports the further development of PREP inhibitors as PP2A activators.
\end{abstract}

\section{Introduction}

Autophagy-lysosomal and ubiquitin-proteasomal pathways are responsible for the degradation of the majority of damaged proteins and their aggregates. Proteasomes degrade unstable, short-lived proteins while long-lived proteins as well as protein aggregates are degraded by macroautophagy (hereafter called autophagy) [1,2]. Changes in autophagy activity are observed in various neurodegenerative disorders, cancer, metabolic and infectious diseases [3,4]. In the case of neurodegenerative proteinopathies such as Parkinson's, Alzheimer's (AD), and Huntington's diseases, accumulation and aggregation of toxic proteins are the main cause of pathology [5]. These toxic protein aggregates can either directly or indirectly inhibit protein degradation pathways that further dampen aggregate clearance from the affected cells $[6,7]$. Additionally, ageing negatively affects protein degradation systems and is considered as one of the greatest risks for neurodegenerative proteinopathies that develop over long period of time and are mainly diagnosed in elderly $[2,8,9]$. Transgenic animals that lack

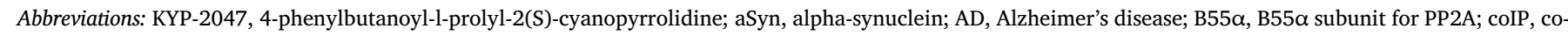

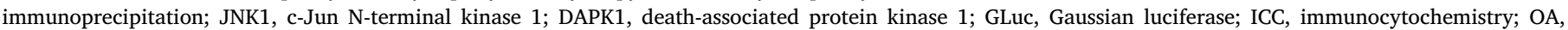

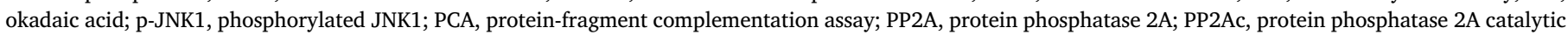

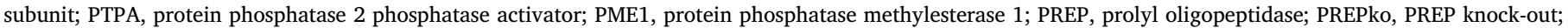

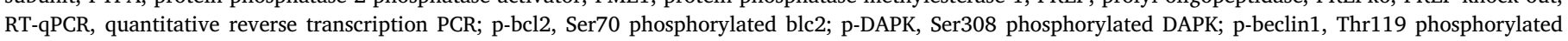
beclin1; p-PP2Ac, Tyr307 phosphorylated PP2Ac; WB, Western blot

* Corresponding author at: Division of Pharmacology and Pharmacotherapy/Drug Research Program, Viikinkaari 5E, P.O. Box 56, 00014 University of Helsinki, Finland.

E-mail address: timo.myohanen@helsinki.fi (T.T. Myöhänen).

${ }^{1}$ Present addresses: R.S.: Seventh affiliated hospital of Sun Yat-Sen university, No.628, Zhenyuan Road, Guangming District, Shenzhen, P.R. China; M.J.: National Institute on Drug Abuse, 251 Bayview Boulevard, Baltimore, MD 21224, USA.
} 
essential autophagy proteins (atg5, atg7, beclin1) develop progressive motor deficits while neuronal cells harbor ubiquitin-positive inclusions that further validates importance of autophagy in aggregate clearance [10-12].

Pharmacological approaches to either upregulate or inhibit autophagy have received a lot of attention. For example, cancer treatment would benefit from either autophagy inhibition or deletion, depending on the cancer type $[4,13]$. While in the case of neurodegenerative disorders, autophagy activation would increase protein aggregate clearance $[3,14]$, and e.g. rapamycin has shown to be effective in reducing behavioral deficits and protein load in the animal models of neurodegenerative diseases $[15,16]$. However, adverse effects have limited the clinical trials of classical autophagy activators [17], and therefore, novel autophagy activating compounds are needed. We have recently identified prolyl oligopeptidase (PREP) inhibitors as novel autophagy inducers, and shown that beclin1-mediated autophagy can be activated by a small-molecule PREP inhibitor, KYP-2047 [18]. Additionally, in aSyn based Parkinson's disease mouse models, PREP inhibition has shown beneficial impact in vitro and in vivo [18-20], and the concept of PREP inhibition was shown to be safe in early clinical trials [21-23].

PREP is a serine protease, and the research on PREP has mainly focused on its enzymatic activity and ability to cleave short prolinecontaining peptides [24]. Hydrolytic action of PREP was the rationale behind the development of small-molecule PREP inhibitors but although showing some beneficial effects in preclinical memory models, their impact on neuropeptide levels in vivo remained unclear [25]. Moreover, physiology of PREP function has remained unclear and since in vivo evidence of PREP cleaving proline-containing peptides is inconsistent, direct protein-protein interactions as we have shown with aSyn [26], might be more relevant for PREP-related actions [27]. Since autophagy has raised interest in drug discovery in several fields, we wanted to study the mechanism of how PREP regulates it. While doing this research, we found that small-molecule PREP inhibitor, KYP-2047, leads to the activation of protein phosphatase 2A (PP2A), and PREP negatively modulates PP2A activity by regulating interactions between catalytic subunit of PP2A (PP2Ac) and its regulatory proteins - protein phosphatase methylesterase 1 (PME1) and protein phosphatase 2 phosphatase activator (PTPA).

\section{Materials and methods}

\subsection{Reagents}

Reagents were purchased from Sigma-Aldrich (St- Louis, MO) if not otherwise specified. Ethanol was purchased from Altia (Helsinki, Finland). The PREP inhibitor, KYP-2047 (4-phenylbutanoyl-1-prolyl2(S)-cyanopyrrolidine), was synthesized for us in the School of Pharmacy, University of Eastern Finland [28].

\subsection{DNA constructs}

Preparation of wt human pAAV1-EF1 $\alpha$-PREP (hPREP; \#59967, Addgene, Brandon Harvey Lab; RRID:Addgene_59967) and pAAV1EF1 $\alpha$-S554A-hPREP (S554A-PREP; \#59968, Addgene, Brandon Harvey Lab; RRID:Addgene_59968) have been described previously in Savolainen et al. [18]. Additional PREP mutants (D149A-PREP, T202CPREP, T204A-PREP, T590C-PREP, and H680A-PREP) with known enzymatic activity and structural changes were selected for site-directed mutagenesis of wt PREP (QuikChange II XL, \#200521, Agilent Technologies).

pAAV-EF1a-hPREP [ $\Delta 189-209 a a]$ (LoopA-PREP) truncate was created by substituting residues from 189 to 209 with a short insert [TGGTQ] [29]. pAAV1-EF1 $\alpha$-PREP was amplified to create two PREP fragments. First fragment contained 1-188aa of PREP with $15 \mathrm{bp} 5^{\prime}$ overhang. Second fragment contained $5^{\prime}$ overhang complementary to first fragment followed by a truncated loop, PREP 210-710aa, and 3' overhang. pAAV1-EF1 $\alpha$-PREP backbone was digested with KpnI-HF (R3142, NEB) and EcoRV-HF (R3195, NEB), gel purified and recombined with inserts using an In-Fusion HD cloning kit (639645, Clontech). Similarly, pAAV-EF1a control vector with 50 bp insert was created by annealing complementary oligonucleotides with $15 \mathrm{bp}$ overhangs (10x annealing buffer: $100 \mathrm{nM}$ Tris $\mathrm{HCl}, 500 \mathrm{mM} \mathrm{NaCl}, 10$ mM EDTA) and recombined with In-Fusion HD cloning kit into the pAAV-EF1a-PREP backbone.

pCMV3-PPP2AC-C-FLAG (HG10420-CF), pCMV3-N-FLAG-PPP2AC (HG10420-NF), pCMV3-PPP2R4A (HG12287-UT), and pCMV3-NFLAG-PPP2R4A (HG12287-NF) were purchased from Sino-Biological. pAAV-EF1a-FLAG(C)-PME1 and pAAV-EF1a-PME1-FLAG(N) inserts were amplified from pUC19-PPME1 (HG29687-U, Sino-Biological) with overhangs containing either $\mathrm{N}$ - or C-terminal FLAG sequence. pAAV1EF1 $\alpha$-PREP backbone was digested with KpnI-HF (NEB) and EcoRV-HF (NEB), gel purified and recombined with all of the aforementioned inserts using an In-Fusion HD cloning kit (Clontech).

For protein-fragment complementation assay (PCA), cDNA of PREP, all of the PREP mutants, PP2Ac, PME1 and PTPA were cloned in a pcDNA3.1/zeo backbone containing humanized G. princeps PCA fragments (GLuc) [27]. All of the GLuc PCA constructs used in this study contained a (GGGGS)2SG linker between the GLuc and cDNA of interest. Based on the N- or C-terminal placement of GLuc fragments, either cDNA targets' start or stop codon was omitted. PREP-GLuc-2N and S554A-PREP-GLuc-2N have been described previously [27]. wt PREP and S554A-PREP constructs and empty GLuc-1N plasmid (backbone) were digested with EcoRI-HF (R3101, NEB) and KpnI-HF (NEB) restriction enzymes, gel purified, and ligated with Ligate-IT Ligation Kit (\#K1422, ThermoFisher Scientific).

PREP mutants (D149A-PREP, T202C-PREP, T204A-PREP, T590CPREP, H680A-PREP and LoopA-PREP), PP2Ac (HG10420-CF, SinoBiological), PME1 (Sino-Biological) and PTPA (HG12287-UT, SinoBiological) cDNA was PCR amplified to create insert oligonucleotides with 15 bp overhangs. Inserts were gel purified and recombined with EcoRI-HF (NEB) and KpnI-HF (NEB) digested and gel purified GLuc-1N, Gluc-2N, GLuc-1C, and GLuc-2C backbone using In-Fusion cloning kit (Clontech). Additionally, pcDNA3.1-PP2Ac and pcDNA3.1-PME1 were created by recombining PCR amplified fragments in GLuc-1N backbone digested with HindIII-HF (R3104, NEB) and EcoRI (NEB) as described above but in this case, the GLuc fragment was removed from the construct. Identity of all constructs was confirmed by DNA sequencing.

\subsection{Cell cultures}

Human embryonic kidney (HEK-293; RRID: CVCL_0045) cells and HEK-293 PREPko cells [30] were cultured in full Eagle's medium (DMEM; \#D6429, Sigma) with an additional $10 \%$ (v/v) fetal bovine serum (FBS; \#16000-044, ThermoFisher Scientific), 1 \% (v/v) L-glutamine-penicillin-streptomycin solution (\#15140122, ThermoFisher Scientific). HEK-293 PREPko cells were cultured in $20 \%$ (v/v) FBS (ThermoFisher Scientific). Human neuroblastoma cells (SH-SY5Y) and SH-SY5Y PREPko cell lines were cultured with Dulbecco's modified eagle medium (DMEM-Glutamax; \#31966021, ThermoFisher Scientific) containing $15 \%$ FBS (ThermoFisher Scientific) for wt and $30 \%$ FBS for SH-SY5Y PREPko cells, $1 \%$ non-essential amino acids (NEAA; \#11140050, ThermoFisher Scientific) and $50 \mu \mathrm{g} / \mathrm{ml}$ Gentamycin (15750-045, ThermoFisher Scientific). Generation of the SH-SY5Y PREPko cells were performed with CRISPR-cas9n plasmid the same way as HEK-293 PREPko cells described in Svarcbahs et al. [30]. Shortly, wt SH-SY5Y were transfected with two CRISPR-cas9n nickase plasmids as described below in cell treatments section and after $48 \mathrm{~h}$ selection single cell clone selection was performed in 96-well plates. Removal of PREP was verified by using PREP activity assay as described earlier [20] (Supplementary Fig. S1).

HEK-293 cells stably expressing GFP-LC3B-RFP construct [31] was 
created by using following protocol. HEK-293 cells were seeded at density of $4 \times 10^{5}$ cells per well on 6-well and transfected with pMRXIP-GFP-LC3-RFP plasmid (2500 ng/well; \#84573, Addgene, Noboru Mizushima Lab; RRID:Addgene_84573) using Lipofectamine 3000 (\#L3000015, ThermoFisher Scientific) transfection reagent according manufacturers protocol. Selection was conducted using $3 \mu \mathrm{g} / \mathrm{ml}$ puromycin for $48 \mathrm{~h}$. After selection cells were trypsinized and seeded on 96-well plate in single cell suspension, and followed based on their GFP expression. Selected cell colonies expressing GFP-LC3B-RFP were cultured further and used in experiments. All cell culture experiments were done between passages 3-20.

\subsection{Animal tissue}

All animal procedures were carried out according to the European Communities Council Directive 86/609/EEC and were approved by the Finnish National Animal Experiment Board. PREPko mice (Deltagene Inc, CA) and wild-type (wt) littermates were-back crossed in C57BL/ 6JRccHsd genetic background (Envigo, The Netherlands; 5-10 back crossings). C57BL/6JRccHsd mouse was used as this was original background for PREPko mouse. Generation of PREPko mice used in these experiments has been described previously [32]. Mice were housed under standard laboratory conditions in National Animal Laboratory Center, University of Helsinki. Cortical tissue samples were collected from naïve 3-4 months old animals (male; weight 25-30 g). No grouping was needed since only mice strains (wt/PREPko) were compared. Briefly, mice were deeply anesthetized with lethal dose of sodium pentobarbital (150 mg/kg; Orion Pharma, Finland) and transcardially perfused with phosphate-buffered saline (PBS, pH 7.4; 137 $\mathrm{mM} \mathrm{NaCl}, 2.7 \mathrm{mM} \mathrm{KCl}, 10 \mathrm{mM} \mathrm{Na}_{2} \mathrm{HPO}_{4}, 1.8 \mathrm{mM} \mathrm{KH}_{2} \mathrm{PO}_{4}$ ). The brains were rapidly frozen in isopentane on dry ice and were kept at $-80{ }^{\circ} \mathrm{C}$ for further analyses. To follow $3 \mathrm{R}$ principle, striatal wt and PREPko mouse tissue samples ( $n=5$ / group) with GFP or PREP overexpression used in this study, were originally prepared and collected for a previous study [33].

\subsection{Cell treatments}

All of the cell transfections were performed with Lipofectamine 3000 (\#L3000015, ThermoFisher Scientific). For co-immunoprecipitation (co-IP) studies, HEK-293 or HEK-293 PREPko cells were plated in $\mathrm{T}-25$ flasks and on the following day they were transfected for $24 \mathrm{~h}$. For transient PREP deletion, cells were plated on 6-well plates $(400,000$ cells per well). On the next day, cells were transfected with PREP CRISPR-cas9 plasmid or empty control plasmid. After $24 \mathrm{~h}$ transfection, cell media was exchanged to one containing puromycin $(1 \mu \mathrm{g} / \mathrm{ml})$ and cells were incubated for an additional $48 \mathrm{~h}$. For PREP inhibitor experiments, either concentration of $10 \mu \mathrm{M}$ (PCA interaction assays) or 1 $\mu \mathrm{M}$ (all other cell culture treatments) of KYP-2047 was used based on our previous studies [18,20,27]. KYP-2047 was diluted to cell culture medium from $100 \mathrm{mM}$ stock in $100 \%$ DMSO, and corresponding concentration of DMSO was used as a vehicle control. Okadaic acid (O8010), a PP2A inhibitor, was diluted to DMSO as $50 \mu \mathrm{M}$ stock and then diluted to $50 \mathrm{nM}$ in cell medium for assays. Bafilomycin $1 \mathrm{~A}$ (SML1661), an autophagy inhibitor, was diluted to 20 or $50 \mathrm{nM}$ from ready made $0.16 \mathrm{mM}$ solution in DMSO.

To determine autophagic flux in 96-well plate format we used slightly modified procedure described in [31]. GFP-LC3-RFP expressing HEK-293 cells were plated on black poly-L-lysine coated 96-well plates at a density of 35,000 cells/well. KYP-2047 was used with concentration $1 \mu \mathrm{M}$ and rapamycin ( $0.5 \mu \mathrm{M}$; BML-A275, Enzo Life Sciences) was used as a positive control for autophagic flux induction and bafilomycin 1A (20-50 nM; SML1661) as autophagy inhibitor. OA was used in GFP-LC3-RFP cells with concentration of $25 \mathrm{nM} .24$ h post treatment cells were washed twice with warm PBS. After washes $150 \mu \mathrm{l}$ PBS was added and GFP signal was read by Victor2 multilabel counter
(PerkinElmer; excitation/emission $485 \mathrm{~nm} / 535 \mathrm{~nm}$ ). Only GFP signal was used in the analysis since RFP signal was not reliably detectable in well-plate format, and in construct RFP may be also degraded by lysosomes to certain extent [31]. However, Hoechst nuclear stain (\#94403) was used to control the total cell count, and this result did not alter results based on GFP-reading. Representative pictures from GFPLC3-RFP expressing cells were taken by Leica DMi8 fluorescence microscope (Leica Microsystems, Wetzlar, Germany).

Autophagy changes were also monitored by using CytoID Autophagy Detection Kit (ENZ-51031, Enzo Life Sciences) according to manufacturer's instructions. Briefly, HEK-293 cells were plated with density of 30,000 cells/well in 96-well plate, and incubated with $24 \mathrm{~h} 1$ $\mu \mathrm{M}$ KYP-2047 +/- $50 \mathrm{nM}$ Bafilomycin A1 while PP242, an mTOR inhibitor, served as a positive control $(0.5 \mu \mathrm{M}$; P0037). GFP signal was read as above and Hoechst nuclear stain was used to correlate GFP signal with cell amount.

\subsection{Western blot}

Cells and animal tissue were lysed in modified RIPA buffer $(50 \mathrm{mM}$ Tris $\mathrm{HCl} \mathrm{pH} \mathrm{7.4,} 1$ \% NP-40, $0.25 \%$ sodium deoxycholate, $150 \mathrm{mM}$ $\mathrm{NaCl}$ ) containing phosphatase inhibitor (\#87786, ThermoFisher Scientific) and protease inhibitor cocktail (\#78430, ThermoFisher Scientific) unless otherwise specified. Samples were sonicated $3 \times 1 \mathrm{~s}$ and centrifuged at $16,000 \mathrm{~g}$ for $15 \mathrm{~min}$. Protein concentration was measured from supernatant with BCA (bicinchoninic acid; \#23225, ThermoFisher Scientific). Standard SDS-PAGE techniques were used and $\sim 30 \mu \mathrm{g}$ of sample was loaded to $12 \%$ (\#4561044, Bio-Rad,) or 4-20 \% Mini-Protean TGX gels (\#4561094, Bio-Rad). Gels were transferred by Trans-Blot Turbo Transfer System (\#1704150, Bio-Rad) onto Trans-Blot Turbo Midi PVDF (\#1704157, Bio-Rad) or nitrocellulose (\#1704159, Bio-Rad) membranes. Membranes were incubated at $+4{ }^{\circ} \mathrm{C}$ overnight in $5 \%$ skim milk or $5 \%$ Bovine Serum Albumin (BSA) in Tris-buffered saline with $0.05 \%$ Tween-20 (TBS-T). A list and details of primary antibodies and respective concentrations is presented in Table 1. After overnight incubation, the membranes were washed and incubated with appropriate HRP-conjugated secondary antibodies for $2 \mathrm{~h}$ in room temperature, goat anti-mouse HRP (\#31430, ThermoFisher Scientific); goat-anti rabbit (\#31463, ThermoFisher Scientific). The membranes containing co-IP samples were incubated with Clean-Blot IP Detection Reagent (\#21230, ThermoFisher Scientific) in 5 $\%$ skim milk. The images were captured using the ChemiDoc XRS + (Bio-Rad, Hercules, CA). To verify that bands are in the linear range of the detection, increasing exposure time and automatic detection of saturated pixels in ImageLab software (version 6.01, Bio-Rad) was used. Thereafter, images were converted to 8-bit grayscale format, and the optical densities (OD) of the bands were measured by ImageJ (histogram area analysis; version 1.48; National Institute of Health, Bethesda, MD). The OD obtained from each band was normalized against the corresponding beta-actin band. The control group each studied protein was set as $100 \%$ for, and treatments were compared to control group of the current protein. To show variance of control group, the control signals were averaged and then control each signal was normalized to this average value. Treatment effects were correlated to average value of control of corresponding protein. All immunoblotting analysis were done with 3-4 technical replicates of each treatment per membrane, and with 2-3 biological replicates (at least 3 different WB assays). All pictures of original WB membranes used for figures in this study are presented in Source file.

\subsection{Co-immunoprecipitation}

Cells were lysed in washing buffer (20 mM Tris, pH 7.5, $150 \mathrm{mM}$ $\mathrm{NaCl}, 1 \mathrm{mM}$ EDTA, 1 mM EGTA, $1 \%$ Triton X-100 and $2.5 \mathrm{mM}$ sodium pyrophosphate) supplemented with protease inhibitor cocktail (\#78430, ThermoFisher Scientific). Samples were sonicated $3 \times 1$ s and 


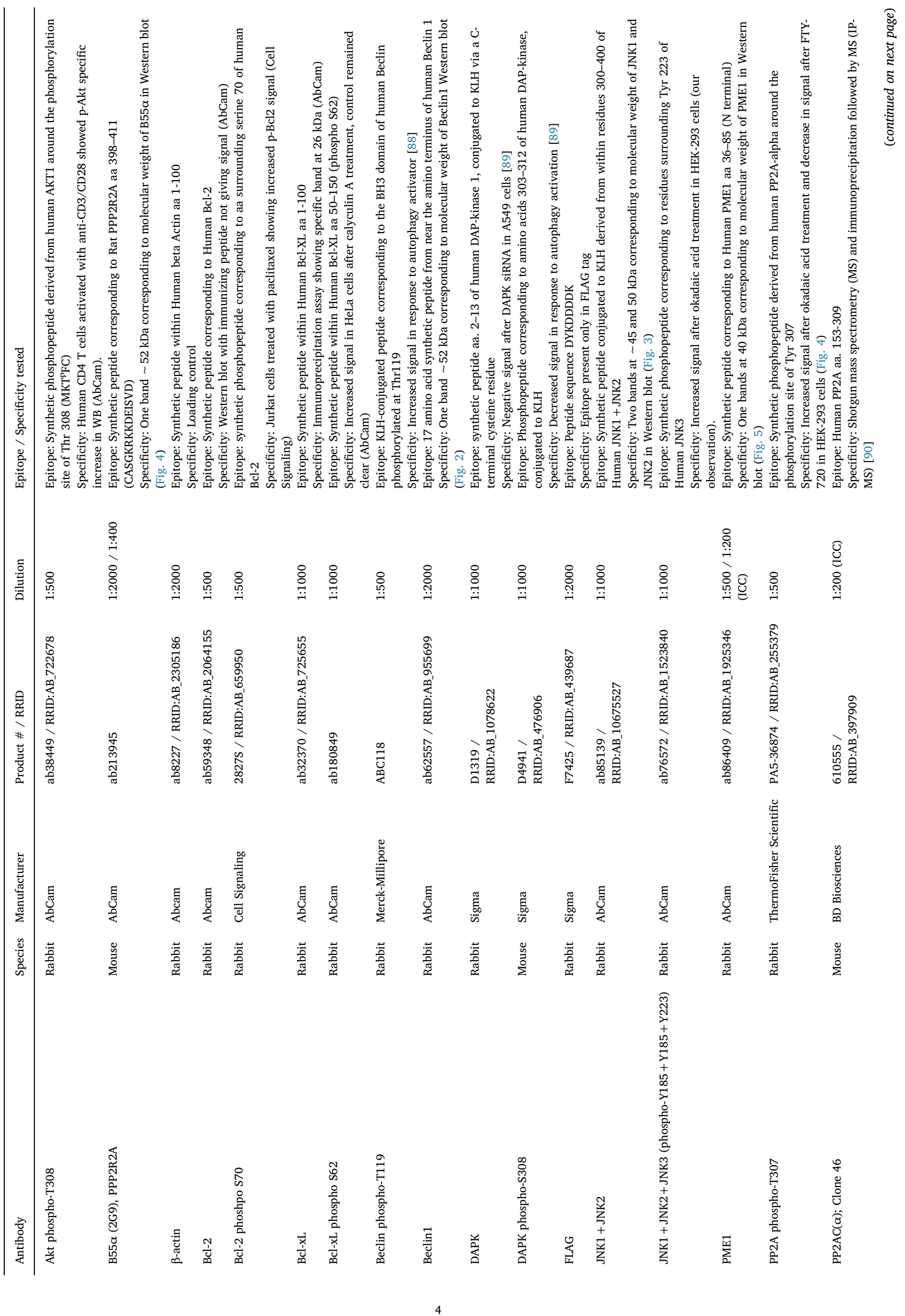


centrifuged at $16,000 \mathrm{~g}$ for $15 \mathrm{~min} .10 \mu \mathrm{L}$ of agarose with anti-FLAG M2 antibody (\#2220, Sigma) was used per immunoprecipitation reaction and were washed according to the manufacturer's instructions. Samples were incubated for $2 \mathrm{~h}$ in washing buffer at $4{ }^{\circ} \mathrm{C}$ with gentle agitation. PREP inhibitor or DMSO control was added directly to the sample at a final concentration of $1 \mu \mathrm{M}$. Beads were washed three times with washing buffer and the FLAG fusion proteins were eluted from the agarose beads with FLAG peptide ( $300 \mu \mathrm{g} / \mathrm{mL}$, \#F3290, Sigma) in Trisbuffered saline for $30 \mathrm{~min}$. Laemmli buffer was added and lysate was boiled for $3 \mathrm{~min}$ at $95{ }^{\circ} \mathrm{C}$, thereafter samples were processed for WB.

\subsection{Reverse transcription $q P C R(R T-q P C R)$}

RNA was extracted using the Aurum Total RNA Mini Kit (\#7326820, Bio-Rad), and cDNA was prepared using iScript Advanced cDNA Synthesis Kit for RT-qPCR (\#1708891, Bio-Rad) with random primers according to the manufacturer's instructions in $20 \mu \mathrm{L}$ reaction. For relative RT-qPCR $1 \mathrm{ng}$ of cDNA was used per reaction in $20 \mu \mathrm{L}$ reaction. PCR was carried out using SsoAdvanced Universal SYBR Green Supermix (\#1725272, Bio-Rad) on a MiniOpticon PCR system (\#3593995, Bio-Rad) with $1 \mathrm{ng}$ of cDNA per reaction with standard cycling conditions $\left(10 \mathrm{~s} 98{ }^{\circ} \mathrm{C} ; 20 \mathrm{~s} 60{ }^{\circ} \mathrm{C}\right.$; $30 \mathrm{~s} 72{ }^{\circ} \mathrm{C}$ for $35-40$ cycles depending on the marker, 5 min extension at $72{ }^{\circ} \mathrm{C}$ and melting curve from $75-95{ }^{\circ} \mathrm{C}$ ). List of primers can be found in Supplementary Table S1. White PCR plates (\#MLL4851, Bio-Rad) with optical flat 8-cap strips (\#TCS0803, Bio-Rad) were used. Three independent experiments and 3 technical replicates per sample were used. MIQE guidelines were followed in RT-qPCR experiments [34].

\subsection{Immunocytochemistry}

ICC to detect changes in co-localization between PP2Ac and PME1, and PP2Ac and PTPA after PREP inhibition or deletion was performed as described in Myöhänen et al. [20]. Briefly, HEK-293 or PREPko HEK293 cells were plated over glass coverslips in a 12-well plate with a density of 100,000 cells/well and allowed to attach overnight. Thereafter, HEK-293 cells were treated for $4 \mathrm{~h}$ with $1 \mu \mathrm{M}$ KYP-2047 or $1 \mu \mathrm{M}$ DMSO (vehicle) and fixed with $4 \%$ paraformaldehyde for staining. Unspecific binding was blocked with $10 \%$ normal goat serum (S-1000, Vector Laboratories) for $30 \mathrm{~min}$ and thereafter cells were incubated with primary antibodies against PP2Ac, PME1 and PTPA overnight at $+4{ }^{\circ} \mathrm{C}$ (details in Table 1 ). After washes, the following secondary antibodies were used to incubate cells $1 \mathrm{~h}$ in room temperature: for mouse PP2Ac, anti-mouse AlexaFluor488 (dilution 1:400; ab150113, Abcam); for rabbit PP2Ac, anti-rabbit AlexaFluor 488 (dilution 1:400; ab150077, Abcam); for mouse PTPA, anti-mouse AlexaFluor 568 (dilution 1:400; ab175473, Abcam); for rabbit PME-1, anti-rabbit AlexaFluor 568 (dilution 1:400; ab175471, Abcam). Cells were mounted with Vectashield containing DAPI to stain nuclei (H-1200, Vector Laboratories). Imaging was performed using Leica TCS SP5 confocal microscope (Leica Microsystems). Images were converted from grayscale to RGB color and recolored with corresponding colors, and minor modifications to brightness and contrast were made.

\subsection{Protein-fragment complementation assay}

The PCA was performed as described previously in Savolainen et al. [27]. HEK-293 or HEK-293 PREPko cells were plated on Poly-L-lysine coated white walled 96-well plates (25,000 cells/well; \#6005070, PerkinElmer) and after $24 \mathrm{~h}$ they were transfected with PCA reporter constructs (100 ng of plasmid DNA/well). $48 \mathrm{~h}$ after transfection, the cells were washed with PBS and phenol red free DMEM (\#21063-029, ThermoFisher Scientific) without serum and antibiotics was added to the cells. The cells were allowed to settle for $1 \mathrm{~h}$. If inhibitors were tested, they were added to the cells in phenol red free DMEM and the cells were treated for $4 \mathrm{~h}$ prior to measurement. A GLuc-PCA signal was 
detected by injecting $25 \mu \mathrm{l}$ of native coelenterazine (Nanolight Technology) to the cells (final concentration $20 \mu \mathrm{M}$ ) and measuring the luminescence signal with Varioskan Flash multiplate reader (ThermoFisher Scientific). All the experiments were done with 8 parallels of each condition and each experiment was repeated at least three times. Transfection efficiency of PCA constructs was assessed by WB.

\subsection{Data and statistical analyses}

All experiments were done at least in triplicate, with 3-8 individual samples on each, and samples were not used to re-analyze same protein. Data are expressed as mean values \pm standard error of the mean (mean \pm SEM), and negative control average was set as $100 \%$ on each assay to reduce variability between repeats. Error bars in the figures represent SEM if not otherwise stated in the figure legend. Differences between groups were analyzed using two-tailed unpaired student's $t$ test. One-way analysis of variance (ANOVA) was followed by Tukey's post-hoc comparison if ANOVA assay gave statistical significance $(\mathrm{P}<0.05)$. Striatal protein levels between GFP and PREP-injected animals were analyzed with a two-way ANOVA followed by Tukey's posthoc comparison if ANOVA assay gave statistical significance $(\mathrm{P}<0.05)$. In all cases, $\mathrm{p}$ values of $<0.05$ were considered to be significant. Statistical analysis was performed using PRISM GraphPad statistical software (version 6.07, GraphPad Software, Inc., San Diego, CA).

\section{Results}

\subsection{PREP inhibition induces autophagic flux}

In our previous studies [18], we have shown that LC3BII levels were increased in cell cultures when PREP was inhibited by KYP-2047, and further increase was observed when KYP-2047 was combined with bafilomycin A1, indicating an increase in autophagic flux [35]. In this study, we tested both 4 and $24 \mathrm{~h}$ PREP inhibition, and interestingly, LC3BII levels were significantly increased in Western blot (WB) at both time points (Fig. 1A). We have also earlier shown that PREP knock-out (PREPko) HEK-293 cells have elevated autophagic flux [30]. At basal conditions, aforementioned cells showed decreased LC3BII levels while PREPko mouse cortical homogenates showed significantly elevated LC3BII levels (Fig. 1B). However, since LC3BII levels alone are not sufficient to verify autophagic flux, we wanted to further characterize this by repeating autophagic flux assay using bafilomycin A1 that blocks the fusion of autophagosomes to lysosomes. When autophagy inducer is used in the presence of bafilomycin A1, it leads to significantly elevated LC3BII levels as autophagosomes are not degreaded [35]. Both in KYP-2047 treated cells (24 h; Fig. 1C) and PREPko HEK293 cells ( 24 h; Fig. 1D), PREP inhibition or removal further increased LC3BII levels when combined with bafilomycin A1. Additionally, we also tested the impact of PREP inhibition on autophagic flux by using GFP-LC3B autophagy reporter cell line and CytoID autophagy detection kit. KYP-2047 showed only mild reduction in GFP signal in autophagy reported cell line but clear increase was seen in CytoID assay, particularly when combined with bafilomycin A1 (Fig. 1A-B). Taken together, these results clearly indicated that both PREP inhibition or removal increase autophagic flux.

\subsection{Impact of PREP modifications on beclin1 and bcl2 phosphorylation and their regulators}

Previously, we observed increase in beclin1 protein levels after PREP inhibition in vitro and in vivo [18]. To initiate autophagy, beclin1 or bcl2/bcl-xL proteins needs to be phosphorylated to reduce complex interaction [36]. 4 h PREP inhibition in HEK-293 cells increased both normal and phosphorylated Thr119 beclin1 (p-beclin1) levels, and also elevated bcl2 levels (Fig. 2A). Even after 24 h PREP inhibition, beclin1, $\mathrm{bcl} 2$, and their corresponding phosphorylated p-beclin and Ser70 blc2 forms (p-bcl2) were significantly increased (supplementary Fig. S1). Similar to PREP inhibitors, PREPko HEK-293 cells had upregulated beclin 1 and p-beclin1 levels but significantly decreased bcl2 levels and elevated p-bcl2 (Fig. 1B), indicating that PREP regulates more beclin1 than bcl2. Transient PREP deletion by CRISPR-Cas9 elevated p-beclin1 and p-bcl2 (supplementary Fig. S1) while in PREPko mouse cortex, bcl$\mathrm{xL}$ levels were lowered and phosphorylated Ser62 bcl-xL was upregulated similar to bcl2/p-bcl2 in PREPko cells (Fig. 2B). Corresponding to these results, PREP overexpression in cells reduced p-beclin1 and bcl2 levels (Fig. 2C). While PREP was restored to PREPko HEK-293 cells, beclin1 phosphorylation decreased but only minor impact on bcl 2 was seen (Fig. 2C), supporting the importance of PREP for beclin1 regulation.

Beclin1 phosphorylation is regulated by death-associated protein kinase 1 (DAPK) [36] while bcl2 is dephosphorylated by c-Jun Nterminal kinase 1 (JNK1) [37]. Therefore, we next studied how PREP modifications affect DAPK and JNK1. Reduced DAPK Ser308 phosphorylation (p-DAPK) activates DAPK [36], and reduced p-DAPK levels were seen in HEK-293 cells after $4 \mathrm{~h}$ KYP-2047 treatment (Fig. 3A). Interestingly, JNK1 phosphorylation (p-JNK1) was also reduced after 4 $\mathrm{h}$ PREP inhibition (Fig. 3A) but this effect was not seen at $24 \mathrm{~h}$ time point for either DAPK or JNK1 (supplementary Fig. S1). In HEK-293 PREPko cells and mouse cortex (Fig. 3B), as well as after transient PREP deletion (supplementary Fig. S1), upregulation in DAPK and JNK1 levels and decrease in p-DAPK and p-JNK1 was significant. When PREP was overexpressed in HEK-293 cells, DAPK levels decreased but p-JNK1 levels were greatly increased (Fig. 3C), and PREP restoration to PREPko cells significantly elevated p-DAPK, JNK1, and p-JNK1 (Fig. 3C), emphasizing the role of PREP in regulation on these kinases. Furthermore, to cross-validate the observation seen in HEK-293 cells, neuroblastoma (SH-SY5Y) cell line was treated for $4 \mathrm{~h}$ with KYP-2047 and we generated a PREPko SH-SY5Y line. Similar changes in DAPK and JNK1 phosphorylation after PREP inhibition or deletion was seen (Supplementary Fig. S1). These findings were further studied in mouse striatal tissue, and when PREP was overexpressed in mouse striatum, increased p-DAPK levels were seen, and similar trend was present when PREP was restored to PREPko mouse striatum (Supplementary Fig. S1). To study if PREP modifications regulate transcription of DAPK or JNK1, we measured the mRNA levels by using quantitative reverse transcription PCR (RT-qPCR). In PREPko cells DAPK mRNA levels were significantly reduced but no changes were detected after PREP inhibition or in PREPko mouse cortex, and no alterations in JNK1 mRNA levels were seen (Supplementary Fig. S3).

\subsection{PP2Ac phosphorylation is decreased after PREP inhibition}

Since PREP inhibition and deletion lead to dephosphorylation of both DAPK and JNK1, we looked for a phosphatase that regulates both of these kinases. Previous studies have reported that PP2A can dephosphorylate both of these kinases [38-40], we wanted to investigate whether PREP inhibition affects PP2A. When we studied the levels of catalytic subunit (PP2Ac) and its inhibitory phosphorylation (Tyr307; p-PP2Ac [41];), we observed slight increase in total PP2Ac and decrease in p-PP2Ac levels after $4 \mathrm{~h}$ (Fig. 4A) and $24 \mathrm{~h}$ (Supplementary Fig. S2) PREP inhibition by $1 \mu \mathrm{M}$ KYP-2047 in HEK-293 cells, indicating PP2A activation. Similar impact was seen in SH-SY5Y cells (Supplementary Fig. S2). Interestingly, rapid changes in phosphorylation levels after 10 min PREP inhibition were not observed (Supplementary Fig. S2), and PP2Ac mRNA levels were not increased at either $4 \mathrm{~h}$ or $24 \mathrm{~h}$ PREP inhibition (supplementary Fig. S3).

To further validate the effect of KYP-2047 on PP2Ac dephosphorylation, HEK-293 cells were incubated for $4 \mathrm{~h}$ with either KYP2047 or $0.5 \mu \mathrm{M}$ FTY720 (fingolimod), a known PP2Ac activator [41,42]. p-PP2Ac levels were decreased at similar extent by KYP-2047 and FTY720 treatments compared to the control group (Fig. 4B). We next analyzed the effect of $4 \mathrm{~h} 50 \mathrm{nM}$ okadaic acid treatment (OA; PP2A 
A
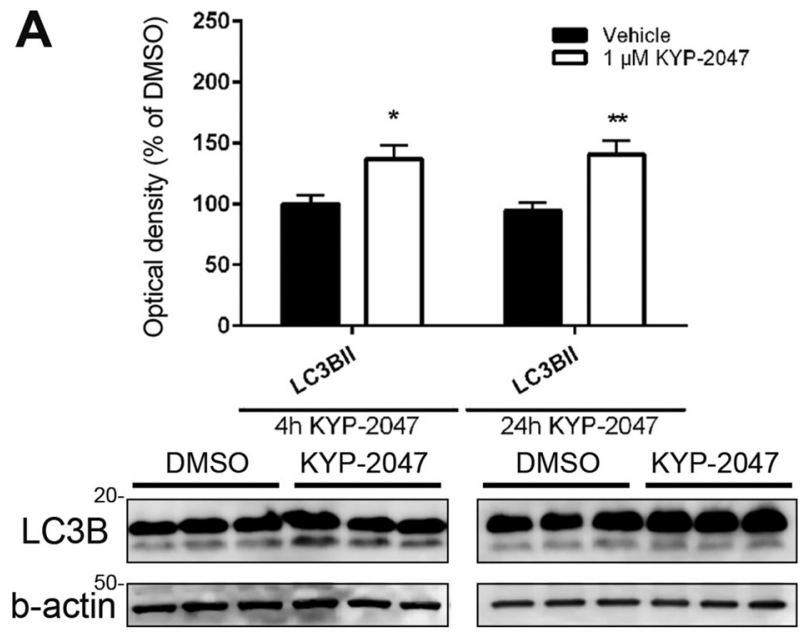

C

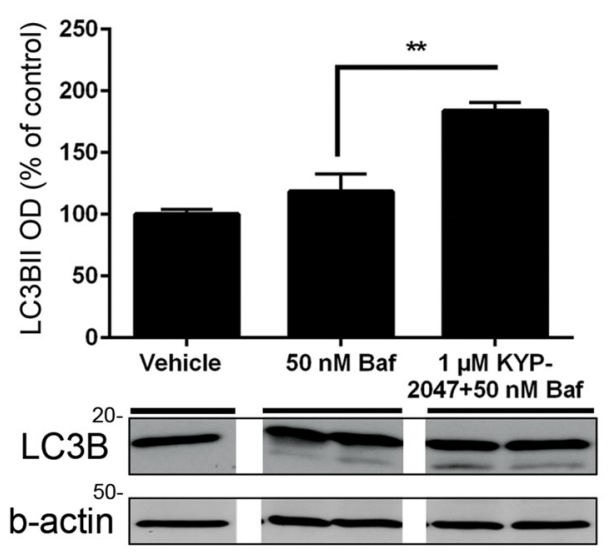

E
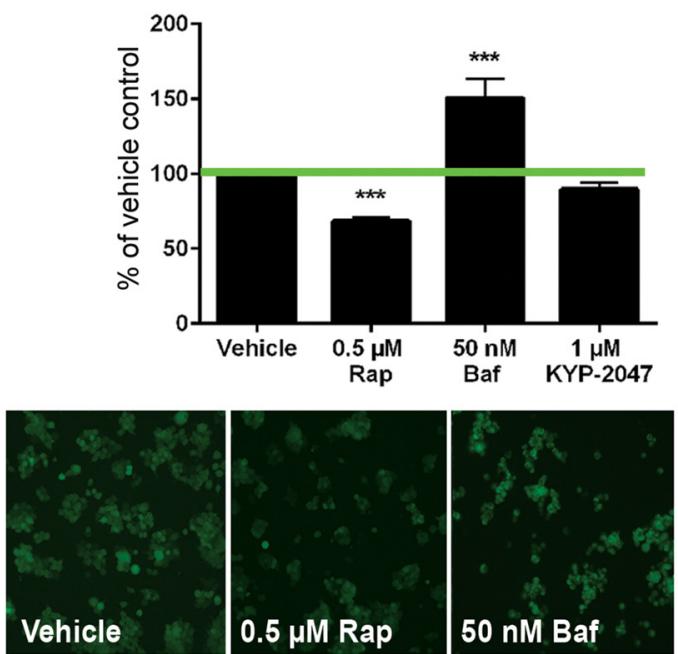

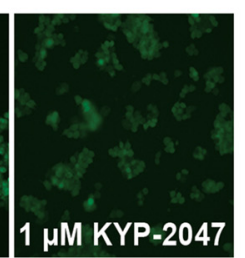

B
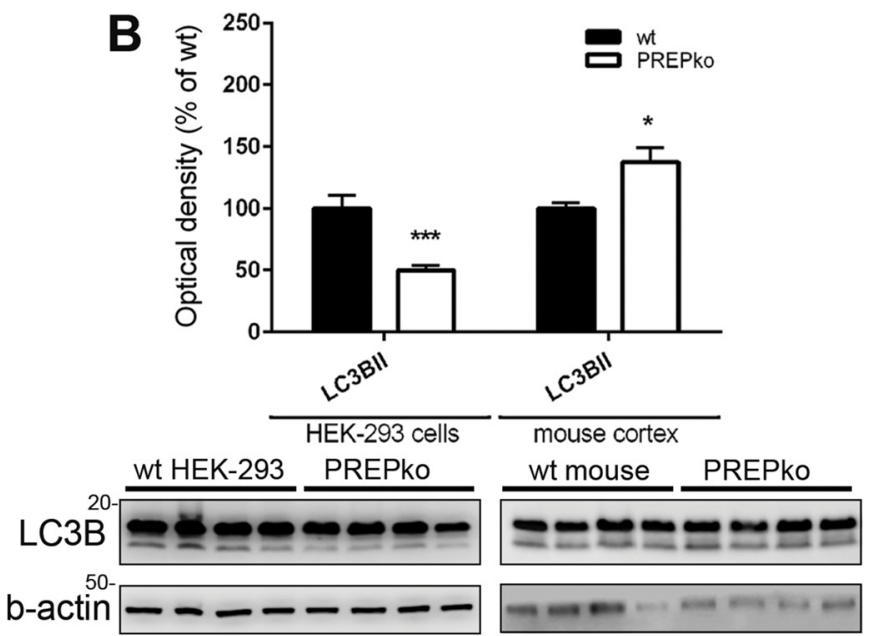

D

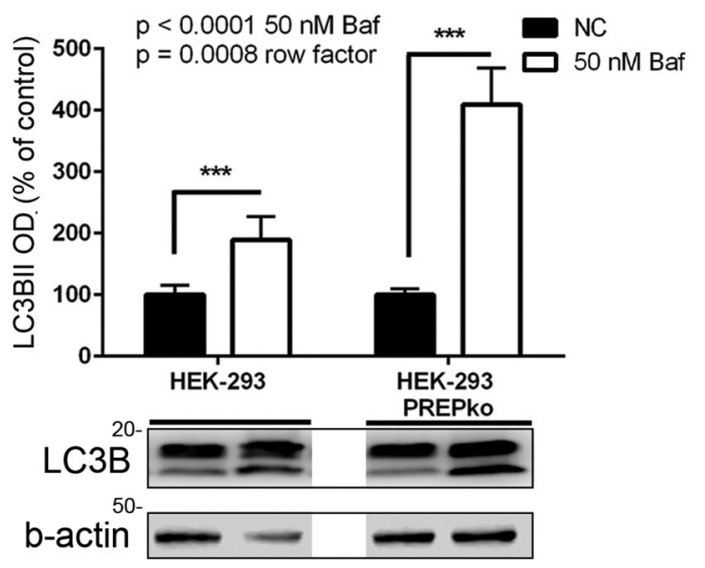

F

CytolD

$\mathrm{NC}$ 口 50 nM Baf

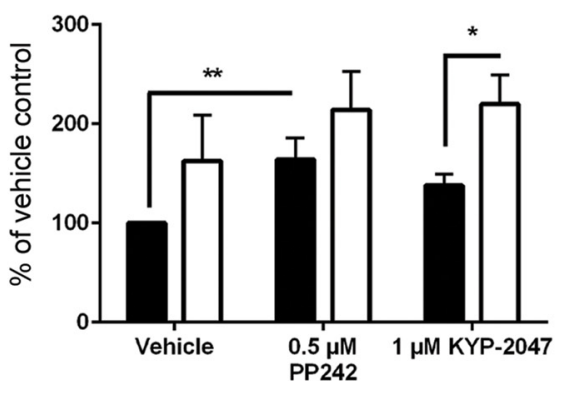

$p=0.000550 \mathrm{nM}$ Baf $p=0.026$ row factor

Fig. 1. PREP inhibition induces autophagic flux. (A) Impact of $4(n=10)$ and $24 \mathrm{~h}(\mathrm{n}=12)$ PREP inhibition by $1 \mu \mathrm{M}$ KYP-2047 on LC3BII levels in HEK-293 cells assayed by Western blot. (B) LC3BII protein levels in PREP knock-out (PREPko) HEK-293 cells $(\mathrm{n}=7$ ) and PREPko mouse cortex ( $\mathrm{n}=10$ ). (C) LC3BII levels after incubating HEK-293 cells with $24 \mathrm{~h}$ vehicle (1 $\mu \mathrm{M}$ DMSO), $50 \mathrm{nM}$ bafilomycin A1 (baf) or $50 \mathrm{nM}$ bafilomycin A1 $+1 \mu \mathrm{M}$ KYP-2047 (n $=8$ ). (D) LC3BII levels after incubating HEK-293 cells or PREPko HEK-293 cells for $24 \mathrm{~h}$ with $50 \mathrm{nM}$ bafilomycin A1 $(\mathrm{n}=6)$ ). (E) GFP readings from GFP-LC3B-RFP stably expressing cell culture after $24 \mathrm{~h}$ exposure to $0.5 \mu \mathrm{M}$ rapamycin (Rap), $50 \mathrm{nM}$ bafilomycin A1 (Baf) and $1 \mu \mathrm{M}$ KYP-2047 with representative pictures from GFP-LC3B-RFP cell cultures after treatments $(\mathrm{n}=7)$. (F) CytoID assay after $24 \mathrm{~h}$ incubations as with GFP-LC3B-RFP cell culture in panel $(0.5 \mu \mathrm{M}$ PP242 as a positive control; $\mathrm{n}=8)$. Data are presented as mean \pm SEM. *, $\mathrm{p}<0.05 ;{ }^{* *}, \mathrm{p}<0.01{ }^{* * *}, \mathrm{p}<0.001$. Unpaired Student's $t$-test in A-B, 1-way ANOVA with Tukey post-test in C and E, 2-way ANOVA with Bonferroni's post-test in $\mathrm{D}(\mathrm{p}<0.000150 \mathrm{nM}$ baf and $\mathrm{p}=0.0008$ cell line) and $\mathrm{F}(\mathrm{p}=0.0005$ baf and $\mathrm{p}=0.026$ treatment). 

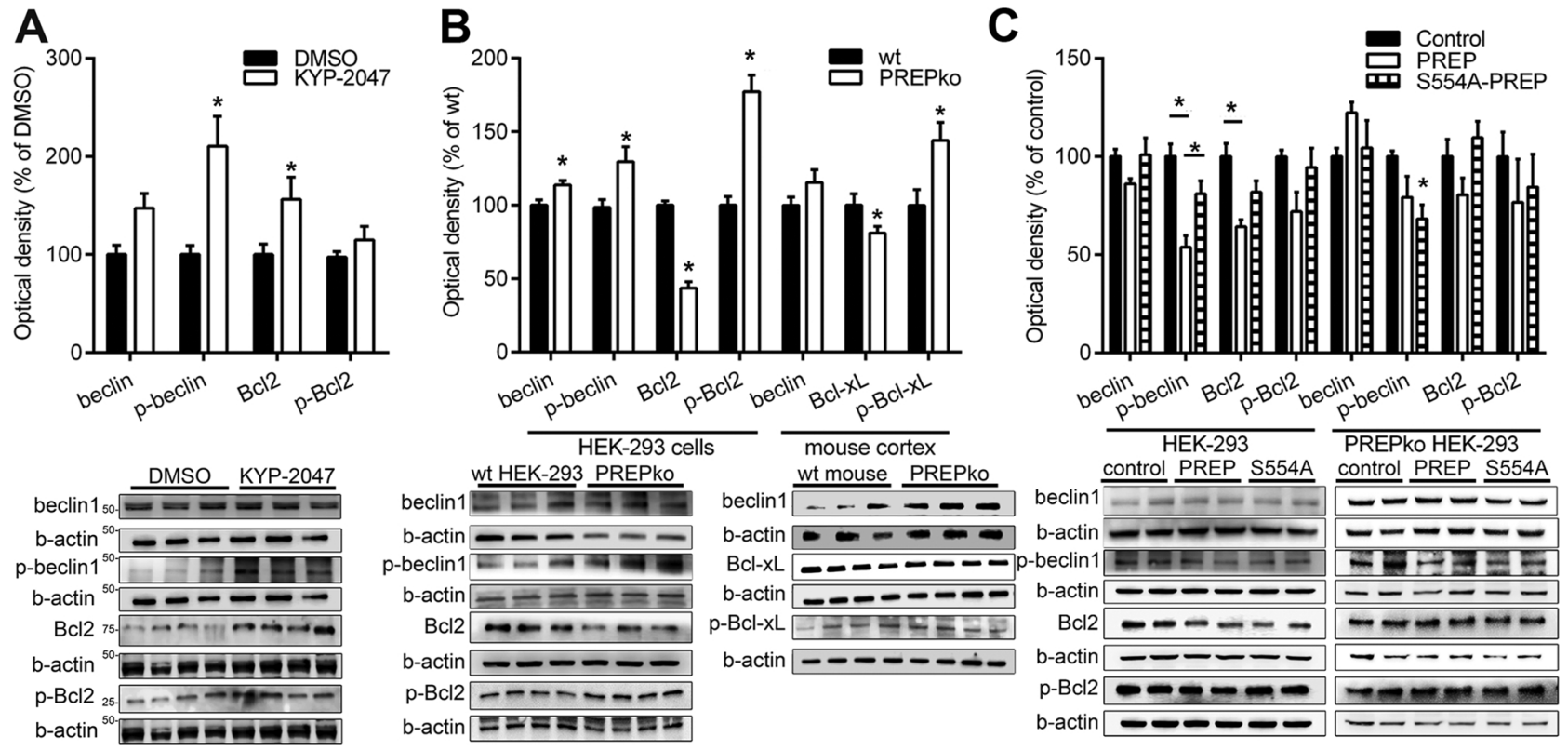

Fig. 2. 4 h PREP modulations affect beclin1 and bcl2/bcl-xl phosphorylation. The levels of beclin1, Thr119 phosphorylated beclin (p-beclin1) and bcl2/Ser70 phosphorylated bcl2 (p-bcl2; cells) or bcl-xl/Ser62 phosphorylated bcl-xl (p-bcl-xl; mouse cortex) were studied by Western blot. (A) HEK-293 cells incubated for $4 \mathrm{~h}$ with a PREP inhibitor (KYP-2047, $1 \mu \mathrm{M} ; \mathrm{n}=8$ ). (B) PREP knock-out (PREPko) HEK-293 cells $(\mathrm{n}=7$ ) and PREPko mouse cortex ( $=7$ ). (C) HEK-293 cells with transient PREP or S554A-PREP (catalytically inactive PREP) overexpression or PREPko HEK-293 cells where PREP or S554A-PREP had been restored (n = 6). Data are presented as mean \pm SEM. *, $\mathrm{p}<0.05$; 1-way ANOVA with Tukey's post-test (C) and unpaired student's $t$-test (A-B).

A
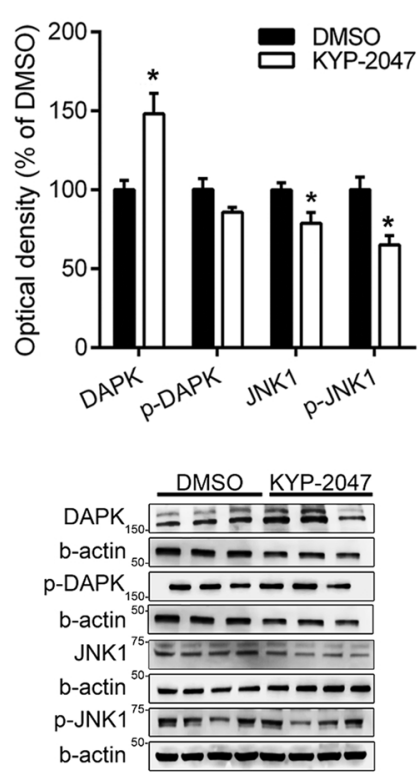

B

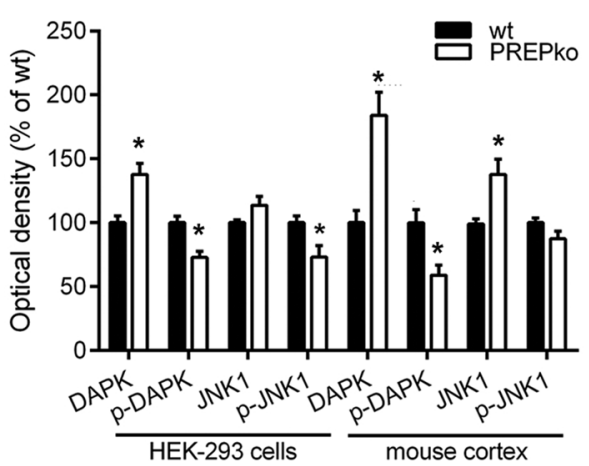

wt HEK-293 PREPko wt mouse PREPko

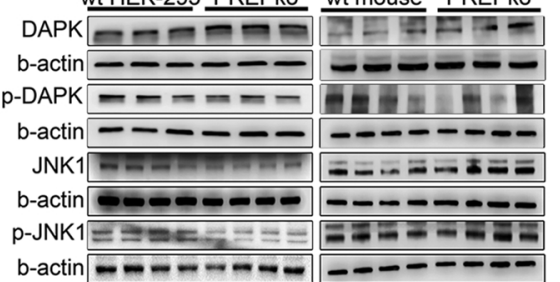

C

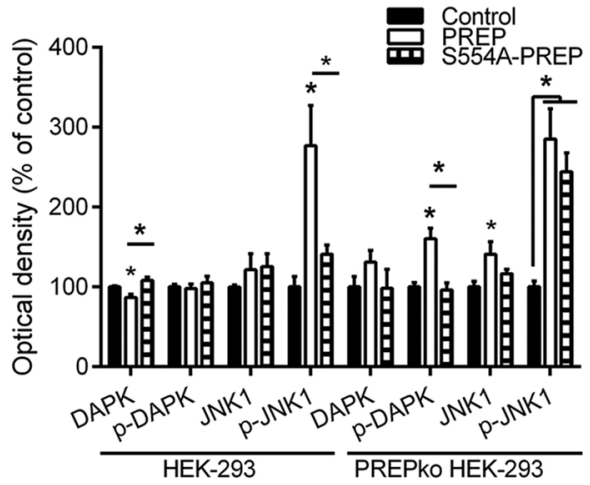

control PREP S554A control PREP S554A

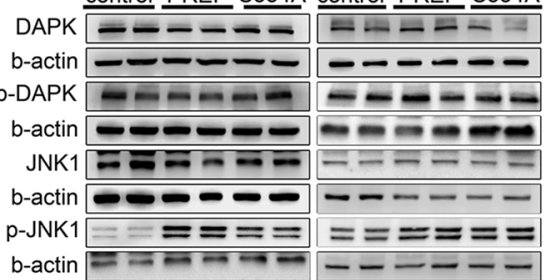

Fig. 3. PREP modulations regulate phosphorylation of DAPK and JNK1. Levels of DAPK and Ser308 phosphorylated DAPK (p-DAPK), and JNK1/Tyr185 phosphorylated JNK1 (p-JNK1) were by immunoblotting. (A) $4 \mathrm{~h}$ with $1 \mu \mathrm{M}$ KYP-2047 in HEK-293 cells ( $\mathrm{n}=13$ ). (B) PREPko HEK-293 cells ( $\mathrm{n}=9$ ) and cortex of PREPko mice $(n=10)$. (C) PREP overexpression in HEK-293 cells and restoring PREP to PREPko HEK-293 cells. Data are presented as mean \pm SEM. *, $p<0.05$; 1 -way ANOVA with Tukey's post-test (C) and unpaired student's $t$-test (A-B).

specific dose [43]) on p-PP2Ac levels in HEK-293 cells in the presence and absence of $1 \mu \mathrm{M}$ KYP-2047 (Fig. 4C). PP2Ac phosphorylation in the OA treated group was significantly elevated as expected. However, the effect was offset in the OA and KYP-2047 co-treated group (Fig. 4C), indicating that KYP-2047 can partially reduce OA inhibition of PP2Ac. Since p-PP2Ac antibody showed decreased signal after FTY720 and increased levels after OA treatments, we concluded that this antibody can be used as an indicator for inactive and active form of PP2Ac.
Deletion of PREP from HEK-293 and SH-SY5Y cells decreased the levels of phosphorylated PP2Ac (Fig. 4D, supplementary Fig. S2) but no changes were seen in HEK-293 or SH-SY5Y PREPko cell PP2Ac protein or mRNA levels (Fig. 4D, supplementary Fig. S3). PREPko mouse cortical tissue had elevated PP2Ac protein and mRNA levels however, changes were not seen in PP2Ac phosphorylation compared to wt littermates (Fig. 4D, supplementary Fig. S3). Additionally, changes were not seen in PP2Ac or its phosphorylation when PREP was either 
A
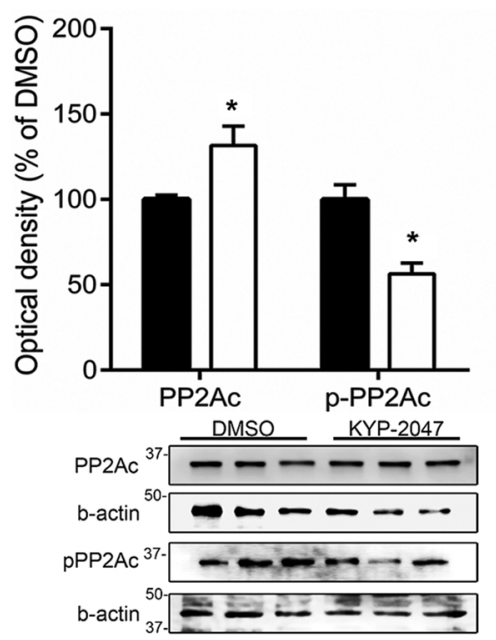

D

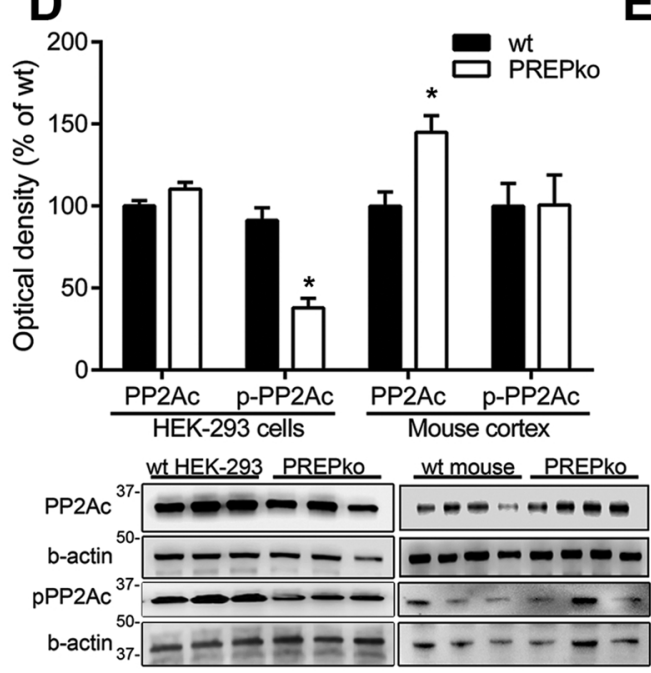

F
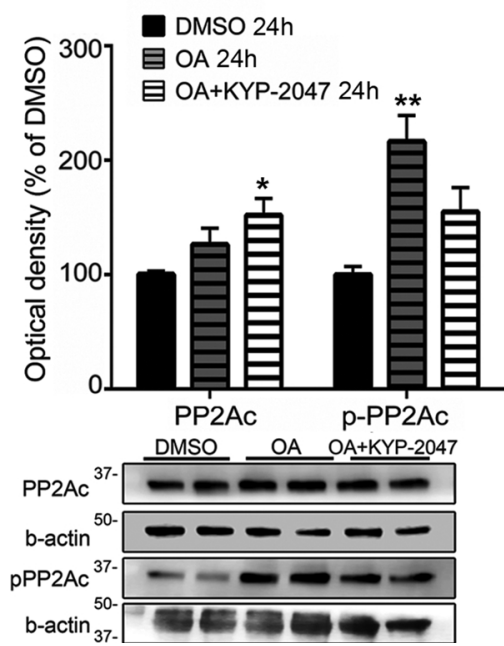

E

G
B

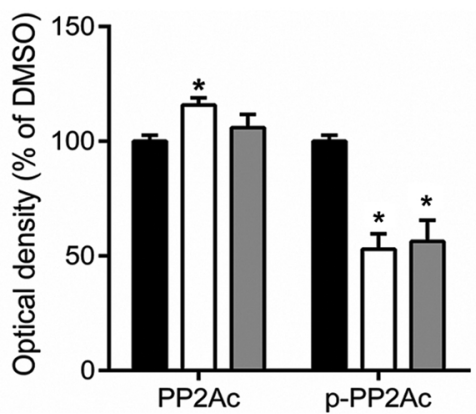

DMSO FTY720 KYP-2047

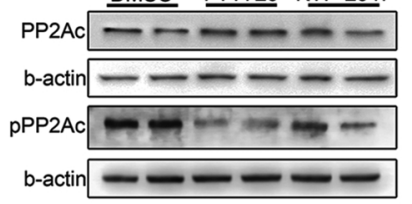

C

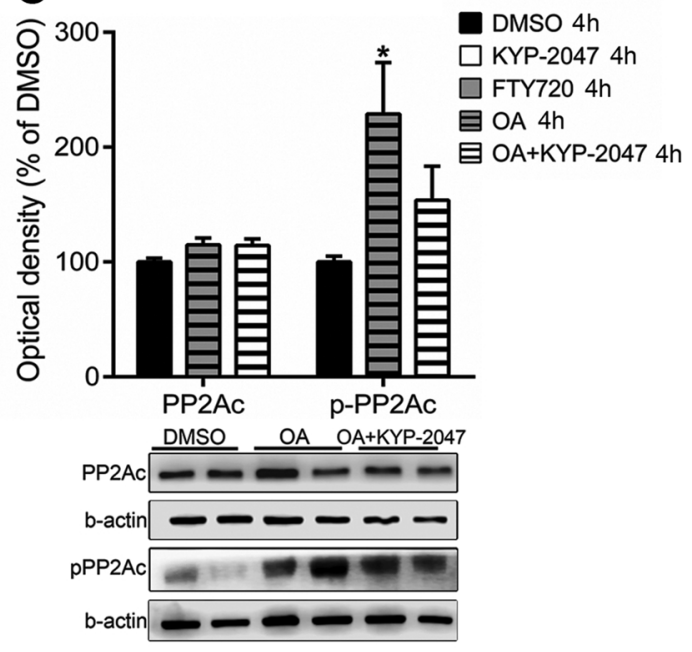

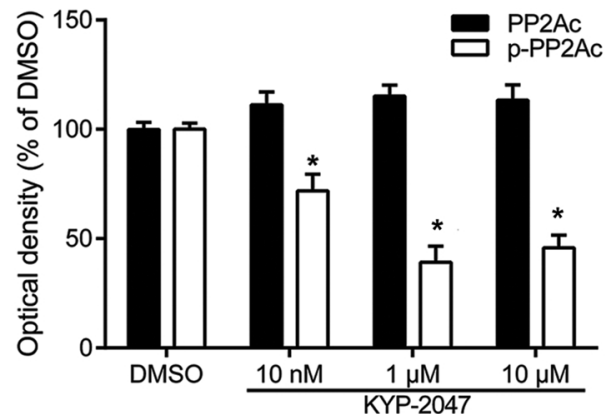

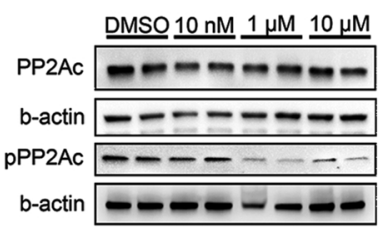

H

GFP-LC3B
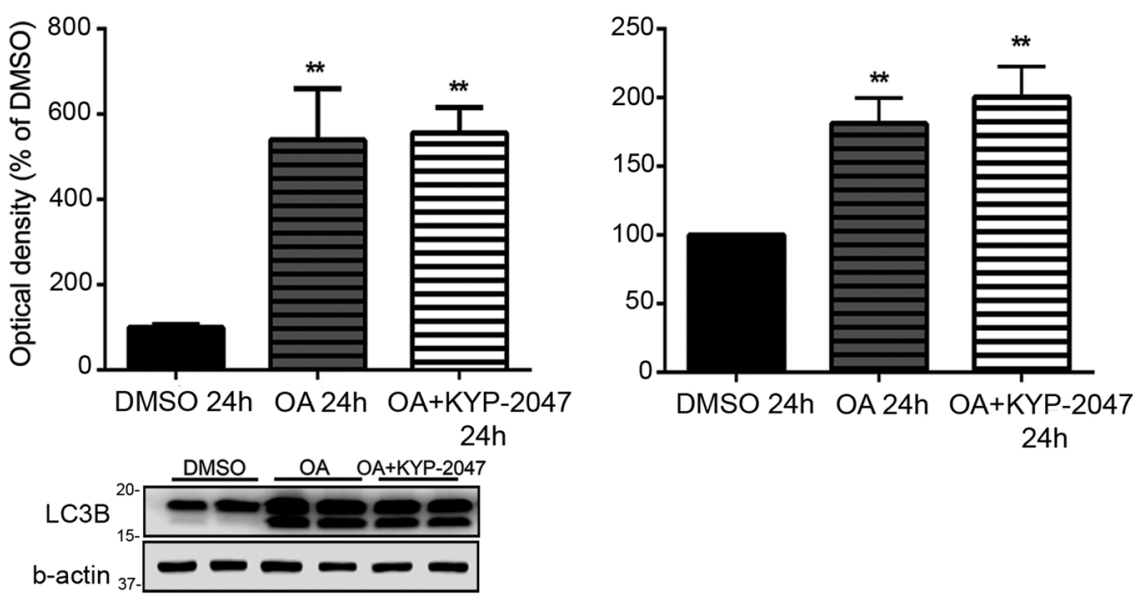

$24 \mathrm{~h}$

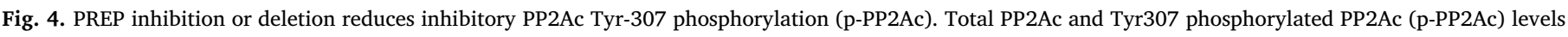

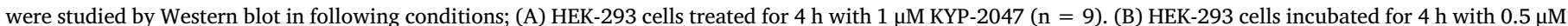

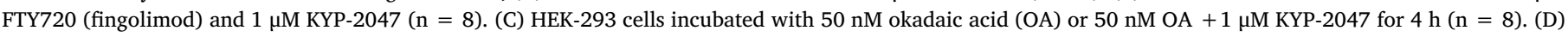

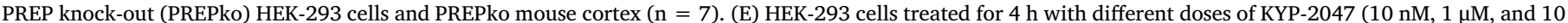

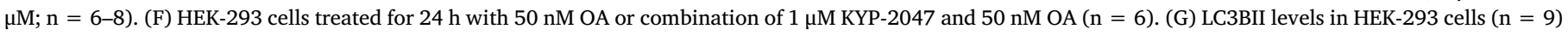

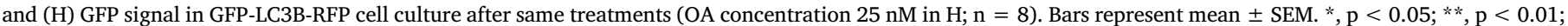
1-way ANOVA with Tukey's post-test (B-C, E-H) and unpaired student's t-test (A, D). 
overexpressed or restored in mouse striatum although PREP overexpression elevated p-PP2Ac levels (supplementary Fig. S2). Fig. 4E demonstrates that PP2A is dephosphorylated dose dependently following KYP treatment without changes in total PP2Ac levels. Full impact is achieved by $1 \mu \mathrm{M}$ KYP-2047 and $10 \mu \mathrm{M}$ dose does not further decrease p-PP2Ac levels (Fig. 4E).

We also wanted to characterize if increased autophagy caused by PREP inhibition is dependent on PP2A activation. We noticed that after $24 \mathrm{~h}$ of OA and KYP-2047 co-treatment, KYP-2047 could attenuate the OA induced PP2A phosphorylation (Fig. 4F). However, LC3BII levels that OA tremendously elevated were not changed when OA was combined with KYP-2047 (Fig. 4G), and the negative impact of OA and OA + KYP-2047 on autophagic flux was confirmed in GFP-LC3B-RFP cell line (Fig. 4H).

\subsection{5-kDa regulatory subunit B alpha (B55 $\alpha$ ), PME-1, and PTPA protein} levels and distribution are altered after PREP inhibition and deletion

After revealing that PREP modulates PP2Ac phosphorylation and activity, we wanted to further clarify mechanisms behind this phenomenon. Due to its important functions in cell, PP2A is highly regulated by interactions with other proteins. PP2A-interacting proteins, such as PME1 and PTPA heavily regulate PP2A complex stability and interaction with regulatory subunits. PME1 is essential for demethylation and inactivation of PP2Ac [44]. At the same time, formation of PP2Ac-PME1 complex increases PP2Ac stability [45] while allowing PTPA to reactivate PP2Ac [46]. We wanted to study how PREP modifications affect aforementioned network and also whether there are changes in $55-\mathrm{kDa}$ regulatory subunit B alpha (B55 $\alpha$ ) since it is responsible for PP2A-Tau interactions [47]. B55 $\alpha$ protein levels were upregulated and PME1 levels were decreased after $4 \mathrm{~h}$ (Fig. 5A) and 24 h KYP-2047 treatment in HEK-293 and in SH-SY5Y cells (Supplementary Fig. S4). Interestingly, PME1 levels were decreased already after 10 min PREP inhibition and after transient PREP deletion while B55 $\alpha$ and PTPA remained unchanged (Supplementary Fig. S54). Meanwhile, no changes in B55a transcription were observed (Supplementary Fig. S3). In PREPko cells and PREPko mouse cortex, B55a protein levels increased relative to control groups (Fig. 5B; Suppelementary Fig. S4). On the contrary, PREP overexpression or restoration reduced B55 $\alpha$ and upregulated PME1 levels in cells (Fig. 5C) and reduced B55 $\alpha$ levels in PREPko mouse striatum, although not significantly (Supplementary Fig. S4). PTPA protein levels were decreased only by PREP overexpression or restoration in wt and PREPko HEK-293 cells (Fig. 5C). Interestingly, inactive PREP (S554A-PREP) overexpression or restoration did not cause any changes in B55 $\alpha$, PME1 or PTPA levels in HEK-293 cells (Fig. 5C).

Since we and others have shown that PREP is able to regulate other proteins via direct protein-protein interactions [27,48,49], we performed immunocytochemistry (ICC) to establish PP2Ac-PME1 and PP2Ac-PTPA colocalization in SH-SY5Y cells with PREP inhibition and deletion (Fig. 5D-E). Colocalization between PP2Ac-PME1 showed increased signal after $4 \mathrm{~h}$ KYP-2047 treatment (Fig. 5D). Notably, PREPko cells exhibited loss of nearly all of the nuclear PME1 and PP2Ac pool. In cytosol, almost all of the PP2Ac-PME1 colocalization was lost and PP2Ac puncta were smaller and diffuse (Fig. 5D). Similarly to PME1, increased colocalization was seen between PP2Ac-PTPA complex after PREP inhibition or deletion (Fig. 5E).

\subsection{PP2A complex forms an interaction with PREP}

The robust change in PP2Ac phosphorylation and changes in PME1 and PTPA levels and colocalizations prompted us to further investigate the interactions between PREP and the PP2Ac complex subunits or regulatory proteins. We performed co-immunoprecipitation (coIP) between FLAG-tagged PP2Ac, PME1 or PTPA and PREP as we had seen changes in their expression after PREP modifications (Fig. 6A-D).
Reverse coIP with FLAG-tagged PREP did not pulldown PP2A complex, however, it has to be emphasized that PREP protein can be linked only from the $\mathrm{N}$-terminal side and any protein anchors from the C-terminal side of PREP renders it inactive [27].

PME1 overexpression increased pulldown of PREP in coIP fractions with either C- or N-terminally FLAG-tagged PP2Ac (Fig. 6A). FLAGPME1 immunoprecipitated PREP (Fig. 6B) but pulldown was not affected by addition of KYP-2047 to the incubation buffer. However, FLAG-PTPA (Fig. 6C) pulldown of PREP was more pronounced when PP2Ac was co-overexpressed. To follow up coIP results, split Gaussian luciferase (GLuc) protein-fragment complementation assay (PCA) was used to screen for putative interaction partners. A luminescence signal indicating a direct interaction between PREP-PP2Ac, PREP-PME1, and PREP-PTPA (Fig. 6D) was seen. These luminescence signals were clearly above background ranging between 10000-200000 relative light units (RLU) depending on the combination of proteins and not all combinations (N or C terminal GLuc fragments) gave a reliable signal. Signals below background level (1000 RLU) were discarded as no interaction (Supplementary Fig. S5). When cells were treated with $10 \mu \mathrm{M}$ KYP2047, the signal intensity was significantly increased for PREP-PME1 and upregulated for PREP-PTPA interaction (Fig. 6D) while negligible changes were seen for the PREP-PP2Ac complex. When PREP or S554APREP complex formation with the aforementioned proteins was tested, signal intensity was increased between S554A-PREP-PME1 and S554APREP-PP2Ac complex (Fig. 6E), but due to the large variation, differences were not significant. In addition, PME1-PP2Ac and PTPA-PP2Ac complex formation was tested to see if PREP restoration or inhibition would have an effect on PP2A subunit interaction (Fig. 6F) but significant changes were not seen.

\subsection{Mutation in the highly mobile PREP Loop B affects PREP interaction} and leads to diminished PREP effect on PP2Ac phosphorylation

Increased PCA signal intensity seen between PP2A complex and S554A-PREP (Fig. 6E) was an indicator that catalytical inactivation of PREP has an impact on PREP interaction with PP2A complex. To test hypothesis that PREP loops are important for not only PREP substrate gating but as well for interaction with other proteins, a set of PREP mutants with earlier published enzymatic activity and partially known kinetics [29,50] were created for PCA screen. D149A is point mutation in $\beta$-propeller, and mutations T202C-PREP (increased activity/mobility) and T204A-PREP (wt activity) are site-mutations located in the Loop A. T590C-PREP mutation is located in the Loop B, and H680A is mutation in the catalytic triad that inactivates PREP hydrolytic activity but still allows inhibitor binding to Ser554 (for more detailed table of PREP point mutations and their impact on PREP functions, see supplementary material in Tsirigotaki et al. [51]). PCA interaction of PREP mutants with PME1 can be seen in the Fig. 7A and PP2Ac or PTPA interaction with PREP in Supplementary Fig. S6. PREP inhibition by KYP-2047 $(10 \mu \mathrm{M})$ significantly upregulated PCA interaction signal between wt PREP, T204A-PREP or H680A-PREP and PME1, PP2Ac or PTPA (Fig. 7A, Supplementary Fig. S6). An outlier observed in all of the PCA tests was T590C mutation in Loop B of PREP; interaction between T590C-PREP and PME1 $(84.87 \% \pm 6.68)$, PP2Ac $(42.63 \% \pm 5.08)$ or PTPA $(35.10 \% \pm 5.62)$ was reduced compared to the wt PREP DMSO control. Particular site-mutation increases the preference for shorter peptides and has been implicated in the substrate gating however, it might be also disrupting the normal function of Loop A [29].

Based on the PCA data, we proceeded to coIP PREP mutants with FLAG-PME1 (Fig. 7B) as PME1 pulldown with PREP has shown the most robust interaction (Fig. 7B). PREP site-mutation did not abolish interaction between PREP and PME1 and all of the tested PREP mutants were immunoprecipitated with FLAG-PME1. However, when a set of PREP mutants were transfected to wt and PREPko HEK-293 cells and tested for the p-PP2Ac levels, an interesting pattern emerged (Fig. 7CD). Wt PREP, S554A-PREP, and T204A-PREP overexpression in the 

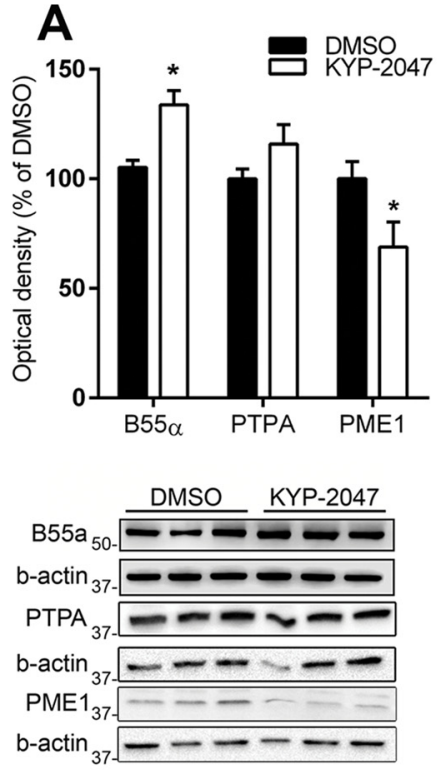

D
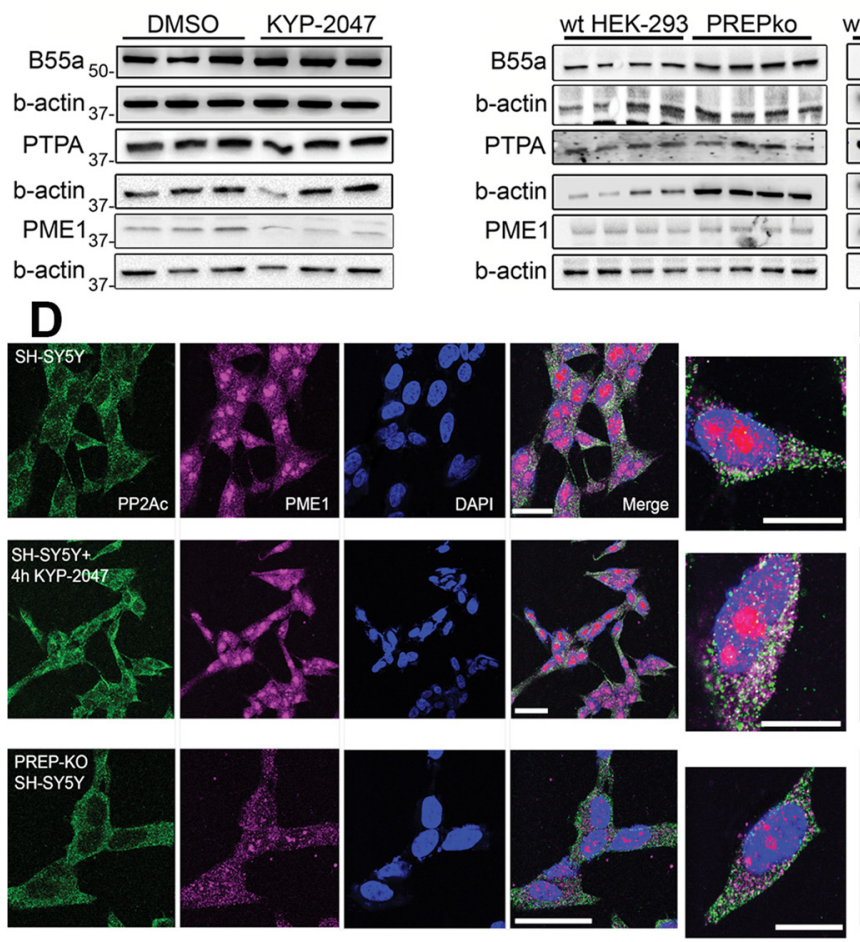
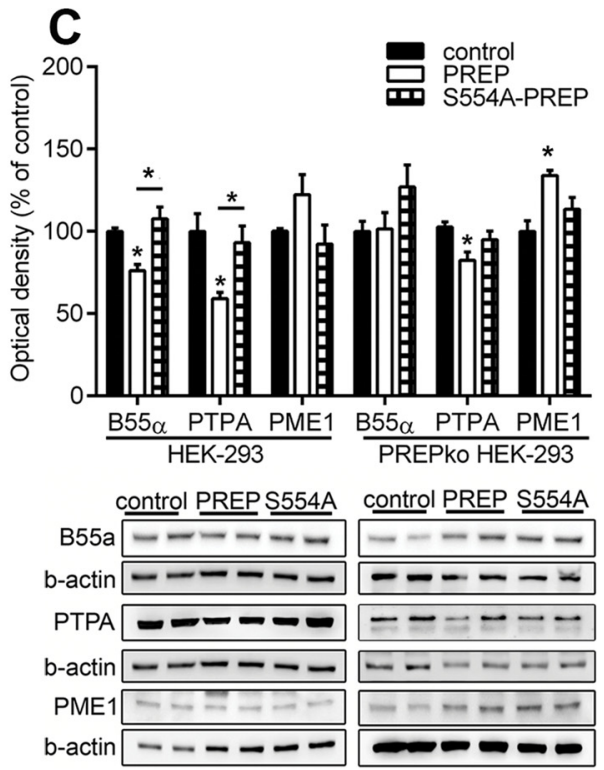

E
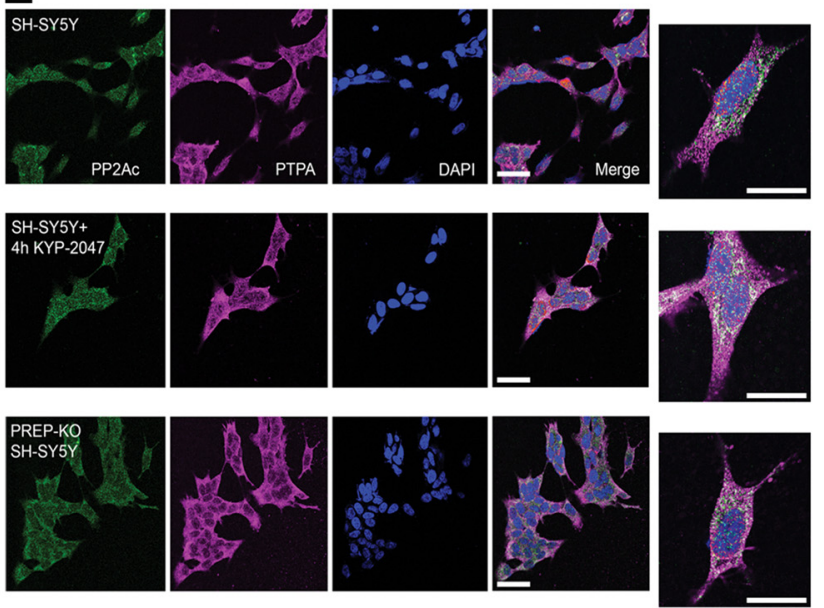

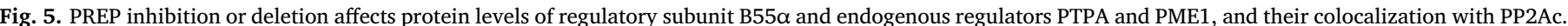

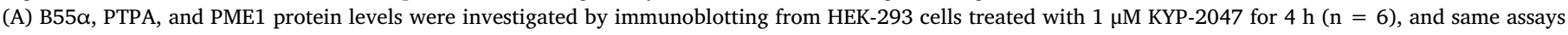

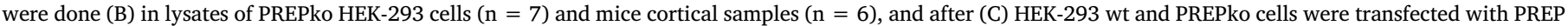

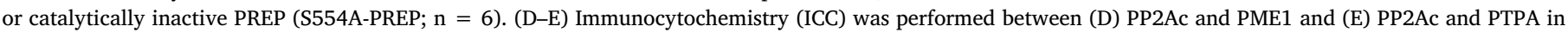

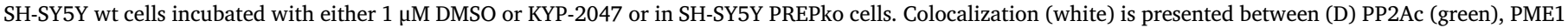

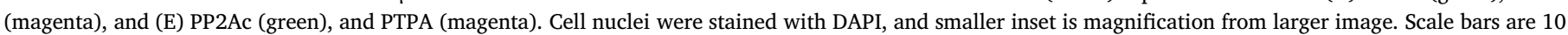

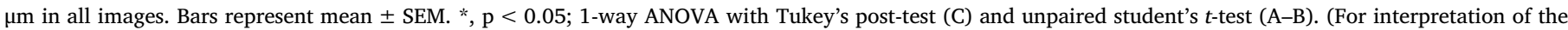
references to colour in this figure legend, the reader is referred to the web version of this article).

PREPko cells resulted in the upregulation of the levels of p-PP2Ac while T590C-PREP mutant overexpression did not result in the upregulation of inhibitory p-PP2Ac levels (Fig. 7D). In wt HEK-293 cells, only overexpression of wt PREP resulted in significant increase in p-PP2A levels (Fig. 7C).

\section{Discussion}

We have earlier shown that PREP can negatively regulate beclin1induced autophagy [18], however the mechanism remained unknown. In this study, we identify PREP as a negative regulator and interaction partner of PP2A complex. This interaction could be modulated by a small-molecule PREP inhibitor that induced a robust dephosphorylation of PP2A, leading to activation of PP2A downstream kinase pathways responsible for the induction of beclin-1 dependent autophagy. Deletion of PREP had similar effect to PREP inhibition while PREP overexpression or restoration inhibited PP2A from cell cultures to in vivo, emphasizing the importance of PREP in PP2A regulation.

PP2A is a major Ser/Thr phosphatase in mammalian cells [52] and main regulator for cell cycle and cell growth. Therefore, its lowered activity and changes in subunit distribution can easily lead to uncontrollable cell proliferation and tumorigenesis [53], tau hyperphosphorylation leading to AD [54], and aggregation of aSyn seen in Parkinson's disease and in dementia with Lewy bodies disease [55]. It is not surprising that activation of PP2A has been suggested as a therapeutic target for several disorders [54,56,57] with multi-target features that might be the key e.g. in finding a disease-modifying therapy for neurodegenerative diseases [42]. Most of the currently known PP2A activators are targeted at cancer treatment where known PP2A activators show tolerable properties [56,58,59], however their relatively high toxicity could be a deterrent for the treatment of neurodegenerative disorders. The concept of PREP inhibition has been shown to be safe in preclinical studies [18-20] and in clinical trials [21-23], and we suggest that the novel PP2A regulatory mechanism revealed in this 

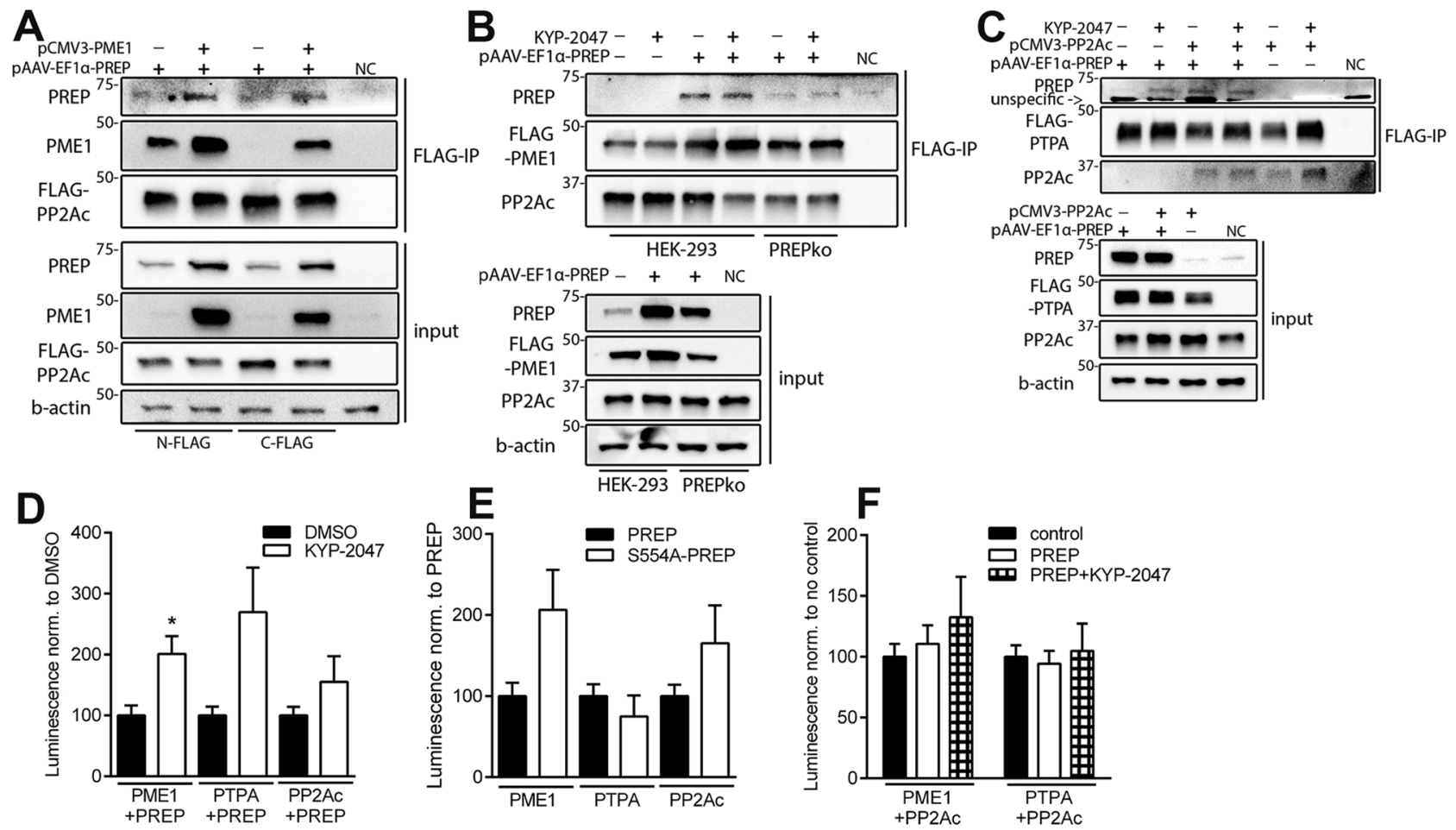

Fig. 6. PREP forms direct protein-protein interactions with PP2A complex and its regulators PME1 and PTPA. (A-D) Co-immunoprecipitation (coIP) followed by immunoblotting was performed to assess the interactions between PP2A complex (PP2Ac, PTPA or PME1) and PREP. (A) PREPko HEK-293 cells were co-transfected with N- or C-terminally FLAG-tagged PP2Ac and PREP, PME1 or empty vector. Co-immunoprecipitation was performed with anti-FLAG antibody (3 independent experiments). (B-C) wt and PREPko HEK-293 cells were transfected with (B) FLAG-PME1 and PREP or empty vector. Lysates were split in two aliquots then immunoprecipitated with FLAG antibody in presence of $1 \mu \mathrm{M}$ KYP-2047 or DMSO. (C) PREPko HEK-293 cells were transfected with FLAG-PTPA, PREP, and PP2Ac or empty vector. Samples were split in aliquots then immunoprecipitated with FLAG antibody in presence of $1 \mu$ M KYP-2047 or DMSO ( $n=3$ ). (D-F) Analyses of the luminescence signal (normalized to corresponding control) from protein-fragment complementation assay (PCA). (D) HEK-293 PREPko cells were co-transfected with Gaussian luciferase (GLuc) fragment fused to PREP and GLuc-PME1, GLuc-PTPA or GLuc-PP2Ac. Prior to analyses, cells were incubated for $4 \mathrm{~h}$ with KYP-2047 (10 $\mu \mathrm{M})$. KYP-2047 treatment significantly increase interaction between PREP and PME1. (E) HEK-293 PREPko cells were co-transfected with GLuc fused PREP or S554APREP and GLuc-PME1, GLuc-PTPA or GLuc-PP2Ac. (F) PCA assay of GLuc fused PP2Ac with GLuc-PME1 or GLuc-PTPA was studied after $4 \mathrm{~h}$ KYP-2047 incubation (10 $\mu \mathrm{M}) . \mathrm{n}=24$ in PCA assays. Bars represent mean \pm SEM. *, $\mathrm{p}<0.05$; PCA data was analyzed by unpaired student's $t$-test (D-F) and one-way ANOVA with Tukey's post-hoc comparison (G).

study offers a potentially safe target to induce PP2A activity, solving a problem that has followed PP2A-targeted drug discovery. It is not completely clear, why PREP inhibition can safely activate PP2A and its downstream pathways but our data indicates that although e.g. autophagy is still active after $24 \mathrm{~h}$ PREP inhibition, the dephosphorylation of critical kinases driving cell towards apoptosis, such as DAPK and JNK1, are restored to basal conditions and this could protect the cell (see below).

There is a growing body of evidence that PREP regulates other proteins via protein-protein interactions, and PREP inhibitors can modify PREP conformation and alter the interaction-based functions $[26,27]$. This is further supported by our current study, where we showed that PREP can regulate complex network between PP2Ac, PME1 and PTPA (Fig. 8), and restoring and overexpressing PREP in cells or in vivo reduced PP2A activity. Interestingly, PREP catalytical activity was not essential for protein binding, supporting the hypothesis that peptidase activity could have secondary effect [60]. However, PP2Ac inhibition and changes in downstream kinase pathways after PREP protein overexpression point to the minute conformation changes and/or PREP enzymatic activity as a prerequisite for the PP2A inactivation. PREP is highly dynamic enzyme, and only porcine PREP can be crystallized without stabilizing the structure with PREP inhibitor $[50,61]$. Its flexible and dynamic external loops control substrate gating $[29,50]$ but we hypothesized that either these loops or the shift in PREP conformation induced by loop movements are responsible for proteinprotein interactions. For the first time, more specific location for regulatory interaction of PREP has been revealed showing that PREP with mutation in loop B (T590C-PREP) exhibit interaction with PME1, PP2Ac, and PTPA but does not upregulate PP2Ac phosphorylation. Therefore, it is possible that loop B or the conformation of PREP that T590C mutation stabilizes is critical for PREP related interactions. More thorough investigation using molecular dynamics is required to clarify this in detail. Additionally, PREP inhibitor binding in the active site and impact of this on loop B dynamics should be studied in order to design novel PREP inhibitors targeted at PP2A activation.

Our results indicate that when PREP is inhibited by KYP-2047, it does not dissolve PP2Ac-PME1 complex but enhances it, which leads to initial decrease in PP2Ac activity. PP2Ac-PME1 complex inhibits PP2Ac catalytical activity and at the same time stabilizes PP2Ac in intracellular pool and protects it from the proteasomal degradation [62]. Interestingly, we also saw reduced PME1 signal in nuclei of PREPko cells. It has been suggested that most of the physiological PME1 effects are dependent on the nuclear PME1 where it is able to stabilize inactive PP2A fraction [63] and it has been suggested that PME1 nuclear accumulation can contribute to PP2A-dependent tumor development $[64,65]$. Therefore, loss of nearly all of the nuclear PME1 and PP2Ac pool would imply PREP role in the nuclear PME1 retention. However, although PREP was interacting with a PP2A inhibitor, PME1, KYP-2047 also increased interaction between PP2Ac and PTPA, leading to its dephosphorylation, activation and holoenzyme formation. Removal of PREP also increased activation of PP2Ac but this is probably due the reduced PP2Ac-PME1 interaction since in PREPko cells almost all of the PP2Ac-PME1 colocalization in cytosol was lost (see schematic drawing in Fig. 8). 


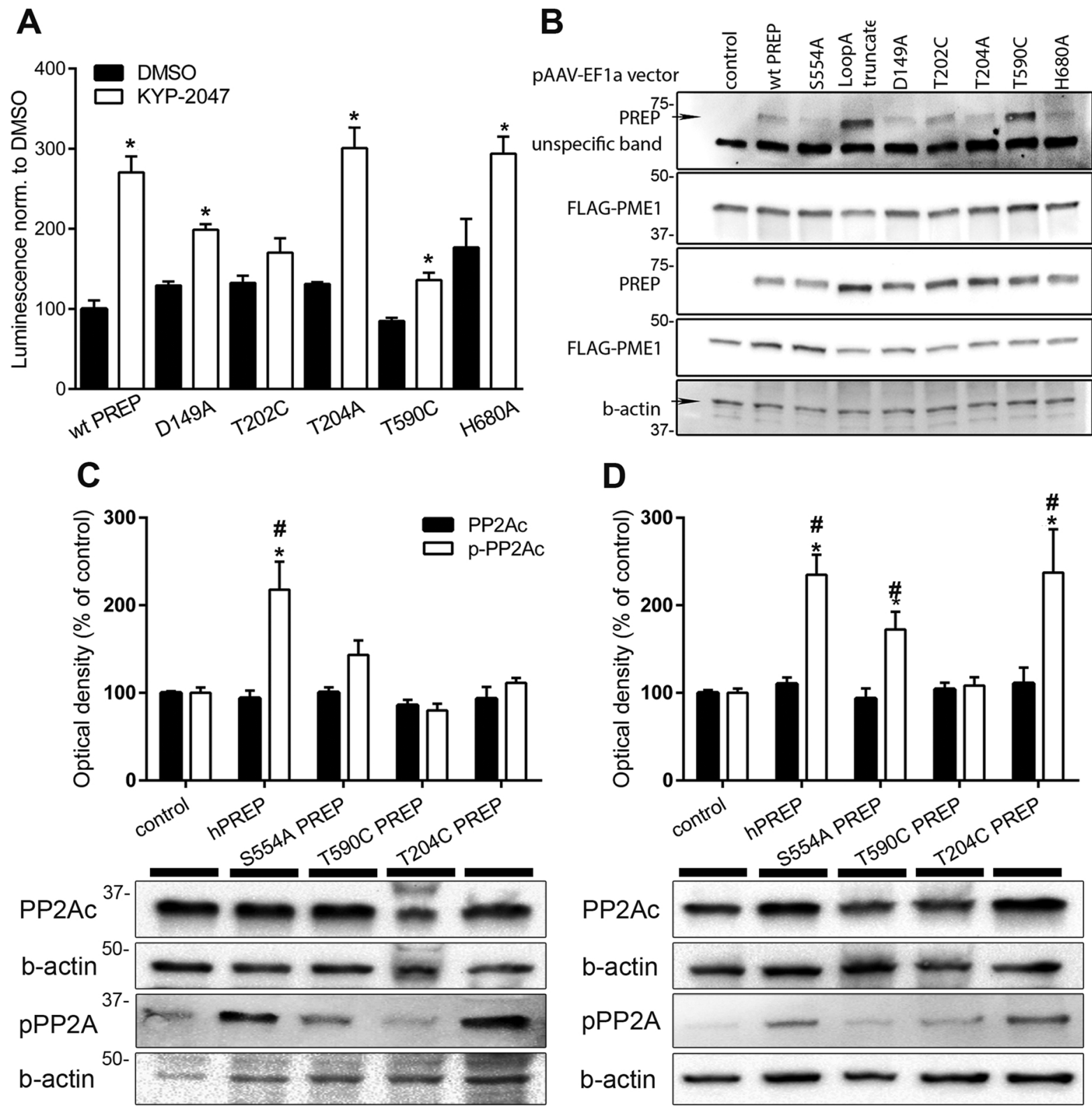

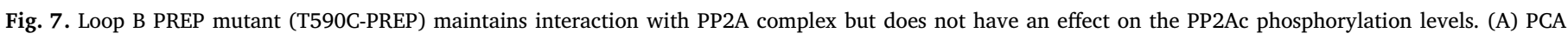

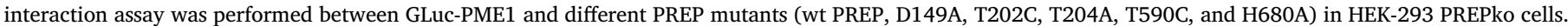

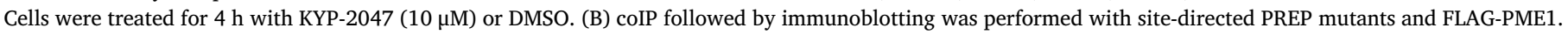

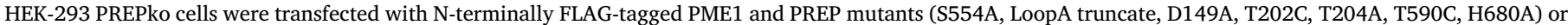

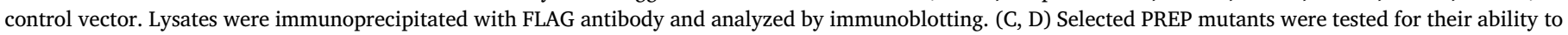

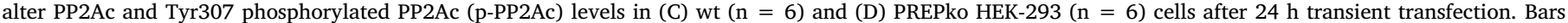

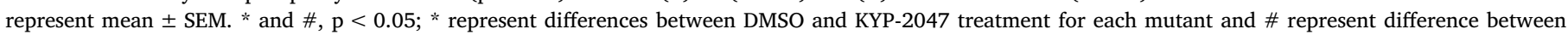

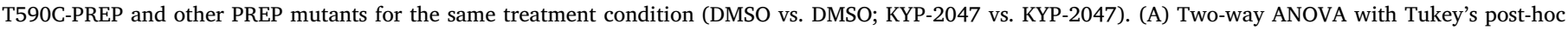
comparison. (B-C) One-way ANOVA with Tukey's post-hoc comparison.

PP2A is known to regulate autophagy via several pathways, including DAPK-beclin1 axis $[36,38,66]$, direct dephosphorylation of ULK1 [67,68], and mTOR regulated interaction with AMBRA [69]. Our results showed that PREP inhibition increased DAPK dephosphorylation at Ser308. PP2A is a known activator of DAPK via dephosphorylation of its amino acid residue while DAPK has been shown to inactivate its catalytical activity by autophosphorylation $[66,70]$. This led to beclin1 phosphorylation that is essential for dissociation of beclin1-bcl2/bcl-xl complex and initiation of autophagy [36]. DAPK regulated autophagy has been mainly associated with autophagy-associated apoptosis [71] but there are no reports about PREP inhibitors inducing apoptosis, rather protecting cell from apoptosis [72]. Our results show that after 24 h PREP inhibition, DAPK phosphorylation was restored to normal levels although tight-binding inhibitor KYP-2047 is inhibiting PREP in cells [20]. We suggest that although PREP inhibition activates critical kinases that can drive cell to apoptosis, PREP inhibition effect is mild and can be overcome by other regulators, preventing cellular death. Additionally, elevated bcl 2 levels induced by PREP inhibition can partially protect cells from apoptosis. Interestingly, PREP inhibition elevated bcl2 levels at $4 \mathrm{~h}$ time point but phosphorylation of bcl2/bcl-xl was increased only after longer PREP inhibition and in PREPko cells and mouse cortex. Elevated bcl2 levels can arise from decreased JNK1 phosphorylation and inactivation [37] that we have shown in this study. Besides, Puttonen et al. [72] reported elevated bcl2 levels in cells 


\section{A. Steady state - inactive but stable PP2Ac}
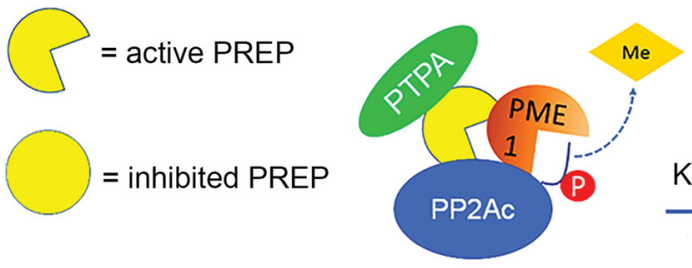

KYP-2047 enhanced

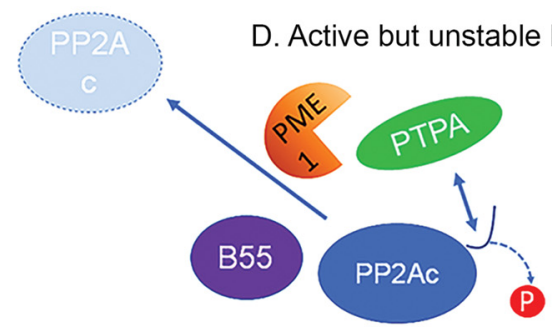

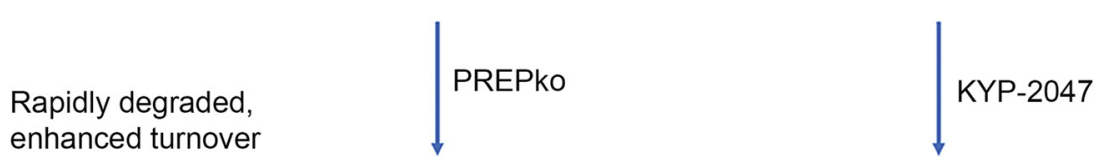

B. Increased inactive intracellular pool of PP2Ac

. PTPA-related activation of PP2Ac

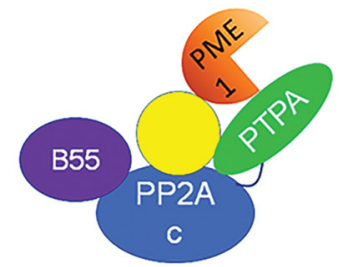

Fig. 8. Schematic representation of possible PREP role in PP2A complex regulation. (A) In normal physiological state, PREP forms an interaction with PP2Ac, PME1 and PTPA, allowing PME1 to demethylate PP2Ac and to protect PP2Ac from rapid degradation [62]. When PREP is inhibited (B), this leads to change in PREP conformation that increases PME1-PP2Ac interaction further increasing the PP2Ac intracellular pool but also moves PME1 from protecting C-tail of PP2Ac, allowing PP2A dephosphorylation at Tyr307, and (C) PTPA interaction with PP2Ac that forms the holoenzyme with B-subunit and activates the phosphatase. (D) If PREP is deleted, this leads to reduced interaction and protection by PME1, leading to increased turnover of PP2Ac but also allows PTPA-related activation that is seen as increased activity. after PREP inhibition but the mechanism remained unclear until now. However, JNK1 was also dephosphorylated and inactivated in PREPko systems where bcl2/bcl-xl phosphorylation was elevated. Earlier study has suggested that JNK1 and other traditional kinases phosphorylating $\mathrm{bcl} 2 / \mathrm{bcl}-\mathrm{xl}$ are not involved in autophagy initiation [73]. This was also shown in our study, and although JNK1 activation is reported to induce autophagy via bcl2/bcl-xl phosphorylation [74], our results indicate that at least in PREP-regulated autophagy, DAPK activation overcomes the impact of JNK1 inactivation in inducing autophagy.

Our finding explains several earlier findings about PREP, including its impacts on cell proliferation, changes in its activity during ontogenesis [75], tumors [76,77], inflammation [78-81] and neurodegeneration [82]. Earlier reports show that PREP activity and protein levels are significantly elevated in tumors and that PREP inhibition affects proliferation rate of certain cancer types namely neuroblastoma, gastric cancer, and human breast cancer cell lines where PP2A activity changes are observed. The effects arise from the $G_{0} / G_{1}$ arrest but the mechanism has remained unclear [76,83]. PP2A is known master regulator for cell cycle [84], and our data indicates that elevated PREP levels can reduce PP2A activity that can contribute to cell division and differentiation during ontogenesis and to tumor growth in cancer. However, it remains unclear what elevates PREP protein levels and activity in cancer. Additionally, PREPko mouse has reduced neuronal size and neuronal connections [32] that may well arise from overactive PP2A [85]. Several studies have connected elevated PREP to inflammation [78,79] but similar to earlier PREP findings, the mechanism has not been revealed. Our data shows strong JNK1 phosphorylation by active PREP, and JNK1 is known the be the main regulator for inflammation, and elevated JNK1 activation has been connected to neurodegeneration [86]. PP2A deficits have also been connected with Tau hyperphosphorylation in AD [54] with increased colocalization between phosphorylated PP2Ac (inactive) and hyperphosphorylated tau in tangle-bearing neurons from AD patients brains [87]. We have earlier shown increased PREP activity and colocalization of PREP with Tau tangles in post mortem AD brains [82], and our current findings further supports the possibility that PREP has a role in pathophysiology of $\mathrm{AD}$ and other tauopathies.

In conclusion, we propose that PREP regulates autophagy by negatively regulating PP2A, and this is the main physiological function for PREP, while hydrolytic function has only secondary purpose. Additionally, increased PREP activity - that has been connected e.g. with tumors and $\mathrm{AD}$ - can lead to reduced PP2A activity, connecting
PREP to pathophysiology of these disorders. As discussed above, the concept of PREP inhibition is considered safe even in clinical trials, and therefore, PREP inhibition can offer a safe possibility for PP2A and autophagy activating therapy that can be applicable for several diseases.

\section{Author contributions}

All authors read and approved the final manuscript. Conceptualization and methodology by R.S., M.J., and T.T.M.; Experiments, R.S., M.J., T.K., U.H.J., L.U., S.O., S.N., and T.T.M.; Writing - Original Draft, R.S.; Writing - Review \& Editing, R.S., M.J., T.K., U.H.J., and T.T.M.; Supervision, M.J. and T.T.M.

\section{Declaration of Competing Interest}

None.

\section{Acknowledgements}

The authors want to thank Dr. Henri Huttunen and Prof. Jukka Westermarck for their comments on the study and manuscript.

\section{Funding}

This study was supported by Academy of Finland (grants 303833, 305710, 267788, and 273799), University of Helsinki 3-year grant, Jane and Aatos Erkko Foundation and Sigrid Juselius Foundation grants for TTM. Funding parties did not have any role in study design, collection, analysis and interpretation of data or in the writing of the report and in the decision to submit the article for publication.

\section{Appendix A. Supplementary data}

Supplementary material related to this article can be found, in the online version, at doi:https://doi.org/10.1016/j.phrs.2019.104558.

\section{References}

[1] N. Mizushima, B. Levine, A.M. Cuervo, D.J. Klionsky, Autophagy fights disease through cellular self-digestion, Nature 451 (2008) 1069-1075, https://doi.org/10. 
1038/nature06639.

[2] D.C. Rubinsztein, The roles of intracellular protein-degradation pathways in neurodegeneration, Nature 443 (2006) 780-786, https://doi.org/10.1038/ nature05291.

[3] D.C. Rubinsztein, P. Codogno, B. Levine, Autophagy modulation as a potential therapeutic target for diverse diseases, Nat. Rev. Drug Discov. 11 (2012) 709-730, https://doi.org/10.1038/nrd3802.

[4] P. Bhat, J. Kriel, B. Shubha Priya, et al., Modulating autophagy in cancer therapy: advancements and challenges for cancer cell death sensitization, Biochem. Pharmacol. 147 (2018) 170-182, https://doi.org/10.1016/j.bcp.2017.11.021.

[5] T.A. Bayer, Proteinopathies, a core concept for understanding and ultimately treating degenerative disorders? Eur. Neuropsychopharmacol. 25 (2015) 713-724, https://doi.org/10.1016/j.euroneuro.2013.03.007.

[6] T.A. Thibaudeau, R.T. Anderson, D.M. Smith, A common mechanism of proteasome impairment by neurodegenerative disease-associated oligomers, Nat. Commun. 9 (2018) 1097, https://doi.org/10.1038/s41467-018-03509-0.

[7] M. Xilouri, O.R. Brekk, N. Landeck, et al., Boosting chaperone-mediated autophagy in vivo mitigates alpha-synuclein-induced neurodegeneration, Brain 136 (2013) 2130-2146, https://doi.org/10.1093/brain/awt131.

[8] T.J. Collier, N.M. Kanaan, J.H. Kordower, Ageing as a primary risk factor for Parkinson's disease: evidence from studies of non-human primates, Nat. Rev. Neurosci. 12 (2011) 359-366.

[9] M.M. Lipinski, B. Zheng, T. Lu, et al., Genome-wide analysis reveals mechanisms modulating autophagy in normal brain aging and in Alzheimer's disease, Proc. Natl. Acad. Sci. 107 (2010) 14164-14169, https://doi.org/10.1073/pnas.1009485107.

[10] T. Hara, K. Nakamura, M. Matsui, et al., Suppression of basal autophagy in neural cells causes neurodegenerative disease in mice, Nature 441 (2006) 885-889, https://doi.org/10.1038/nature04724.

[11] M. Komatsu, S. Waguri, T. Chiba, et al., Loss of autophagy in the central nervous system causes neurodegeneration in mice, Nature 441 (2006) 880-884, https://doi. org/10.1038/nature04723.

[12] S. Sato, T. Uchihara, T. Fukuda, et al., Loss of autophagy in dopaminergic neurons causes Lewy pathology and motor dysfunction in aged mice, Sci. Rep. 8 (2018) 2813, https://doi.org/10.1038/s41598-018-21325-w.

[13] G.J. Yoshida, Therapeutic strategies of drug repositioning targeting autophagy to induce cancer cell death: from pathophysiology to treatment, J. Hematol. Oncol. 10 (2017) 67, https://doi.org/10.1186/s13045-017-0436-9.

[14] J. Nah, J. Yuan, Y.K. Jung, Autophagy in neurodegenerative diseases: from mechanism to therapeutic approach, Mol. Cells 38 (2015) 381-389, https://doi.org/ 10.14348/molcells.2015.0034.

[15] J. Bové, M. Martínez-Vicente, M. Vila, Fighting neurodegeneration with rapamycin: mechanistic insights, Nat. Rev. Neurosci. 12 (2011) 437, https://doi.org/10.1038/ nrn3068.

[16] Z. Cai, L.-J. Yan, Rapamycin, autophagy, and alzheimer's disease, J. Biochem. Pharmacol. Res. 1 (2013) 84-90.

[17] B. Levine, M. Packer, P. Codogno, Development of autophagy inducers in clinical medicine, J. Clin. Invest. 125 (2015) 14-24, https://doi.org/10.1172/JCI73938.

[18] M.H. Savolainen, C.T. Richie, B.K. Harvey, et al., The beneficial effect of a prolyl oligopeptidase inhibitor, KYP-2047, on alpha-synuclein clearance and autophagy in A30P transgenic mouse, Neurobiol. Dis. 68 (2014) 1-15, https://doi.org/10.1016/j. nbd.2014.04.003.

[19] R. Svarcbahs, U.H. Julku, T.T. Myöhänen, Inhibition of prolyl oligopeptidase restores spontaneous motor behavior in the alpha-synuclein virus vector-based parkinson's disease mouse model by decreasing alpha-synuclein oligomeric species in mouse brain, J. Neurosci. 36 (2016) 12485-12497, https://doi.org/10.1523/ JNEUROSCI.2309-16.2016.

[20] T.T. Myöhänen, M.J. Hannula, R. Van Elzen, et al., A prolyl oligopeptidase inhibitor, KYP-2047, reduces alpha-synuclein protein levels and aggregates in cellular and animal models of Parkinson's disease, Br. J. Pharmacol. 166 (2012) 1097-1113 10.1111/j.1476-5381.2012.01846.x.

[21] P. Morain, J.L. Robin, G. De Nanteuil, et al., Pharmacodynamic and pharmacokinetic profile of S 17092, a new orally active prolyl endopeptidase inhibitor, in elderly healthy volunteers. A phase I study, Br. J. Clin. Pharmacol. 50 (2000) 350-359.

[22] K. Umemura, K. Kondo, Y. Ikeda, et al., Pharmacokinetics and safety of JTP-4819, a novel specific orally active prolyl endopeptidase inhibitor, in healthy male volunteers, Br. J. Clin. Pharmacol. 43 (1997) 613-618.

[23] K. Umemura, K. Kondo, Y. Ikeda, et al., Pharmacokinetics and safety of Z-321, a novel specific orally active prolyl endopeptidase inhibitor, in healthy male volunteers, J. Clin. Pharmacol. 39 (1999) 462-470.

[24] A. Moriyama, M. Nakanishi, M. Sasaki, Porcine muscle prolyl endopeptidase and its endogenous substrates, J. Biochem. 104 (1988) 112-117.

[25] P.T. Männistö, J.I. Venäläinen, A.J. Jalkanen, J.A. Garcia-Horsman, Prolyl oligopeptidase: a potential target for the treatment of cognitive disorders, Drugs News Prespect. 20 (2007) 293-305.

[26] I. Brandt, M. Gerard, K. Sergeant, et al., Prolyl oligopeptidase stimulates the aggregation of alpha-synuclein, Peptides 29 (2008) 1472-1478, https://doi.org/10. 1016/j.peptides.2008.05.005.

[27] M.H. Savolainen, X. Yan, T.T. Myöhänen, H.J. Huttunen, Prolyl oligopeptidase enhances alpha-synuclein dimerization via direct protein-protein interaction, $\mathrm{J}$. Biol. Chem. 290 (2015) 5117-5126, https://doi.org/10.1074/jbc.M114.592931.

[28] E.M. Jarho, J.I. Venalainen, J. Huuskonen, et al., A cyclopent-2-enecarbonyl group mimics proline at the $\mathrm{P} 2$ position of prolyl oligopeptidase inhibitors, J. Med. Chem. 47 (2004) 5605-5607, https://doi.org/10.1021/jm049503w.

[29] Z. Szeltner, T. Juhász, I. Szamosi, et al., The loops facing the active site of prolyl oligopeptidase are crucial components in substrate gating and specificity,
Biochimica et Biophysica Acta (BBA) - Proteins Proteomics 1834 (2013) 98-111, https://doi.org/10.1016/j.bbapap.2012.08.012.

[30] R. Svarcbahs, U.H. Julku, S. Norrbacka, T.T. Myöhänen, Removal of prolyl oligopeptidase reduces alpha-synuclein toxicity in cells and in vivo, Sci. Rep. 8 (2018) 1552, https://doi.org/10.1038/s41598-018-19823-y.

[31] T. Kaizuka, H. Morishita, Y. Hama, et al., An autophagic flux probe that releases an internal control, Mol. Cell 64 (2016) 835-849, https://doi.org/10.1016/j.molcel. 2016.09.037.

[32] C. Höfling, N. Kulesskaya, K. Jaako, et al., Deficiency of prolyl oligopeptidase in mice disturbs synaptic plasticity and reduces anxiety-like behaviour, body weight, and brain volume, Eur. Neuropsychopharmacol. 26 (2016) 1048-1061, https://doi. org/10.1016/j.euroneuro.2016.02.015.

[33] U.H. Julku, A.E. Panhelainen, S.E. Tiilikainen, et al., Prolyl oligopeptidase regulates dopamine transporter phosphorylation in the nigrostriatal pathway of mouse, Mol. Neurobiol. 55 (2018) 470-482, https://doi.org/10.1007/s12035-016-0339-8.

[34] S.A. Bustin, V. Benes, J.A. Garson, et al., The MIOE guidelines: minimum information for publication of quantitative real-time PCR experiments, J. Clin. Chem. 55 (2009) 611-622 10.1373/clinchem.2008.112797 \%J Clinical Chemistry.

[35] D.J. Klionsky, K. Abdelmohsen, A. Abe, et al., Guidelines for the use and interpretation of assays for monitoring autophagy (3rd edition), Autophagy 12 (2016) 1-222, https://doi.org/10.1080/15548627.2015.1100356.

[36] E. Zalckvar, H. Berissi, M. Eisenstein, A. Kimchi, Phosphorylation of Beclin 1 by DAP-kinase promotes autophagy by weakening its interactions with Bcl-2 and BclXL, Autophagy 5 (2009) 720-722, https://doi.org/10.4161/auto.5.5.8625.

[37] X. Deng, L. Xiao, W. Lang, et al., Novel role for JNK as a stress-activated Bcl2 kinase, J. Biol. Chem. 276 (2001) 23681-23688, https://doi.org/10.1074/jbc. M100279200.

[38] N. Fujiwara, T. Usui, T. Ohama, K. Sato, Regulation of beclin 1 protein phosphorylation and autophagy by protein phosphatase 2A (PP2A) and death-associated protein kinase 3 (DAPK3), J. Biol. Chem. 291 (2016) 10858-10866, https://doi. org/10.1074/jbc.M115.704908.

[39] R.C. Widau, Y. Jin, S.A. Dixon, B.E. Wadzinski, P.J. Gallagher, Protein phosphatase 2A (PP2A) holoenzymes regulate death-associated protein kinase (DAPK) in ceramide-induced anoikis, J. Biol. Chem. 285 (2010) 13827-13838, https://doi.org/ 10.1074/jbc.M109.085076.

[40] T.P. Shanley, N. Vasi, A. Denenberg, H.R. Wong, The Serine/Threonine phosphatase, PP2A: endogenous regulator of inflammatory cell signaling, J. Reprod. Immunol. 166 (2001) 966-972, https://doi.org/10.4049/jimmunol.166.2.966.

[41] J. Chen, B. Martin, D. Brautigan, Regulation of protein serine-threonine phosphatase type-2A by tyrosine phosphorylation, Science 257 (1992) 1261-1264 10.1126/ science.1325671\%

[42] R. Baskaran, B.K. Velmurugan, Protein phosphatase $2 \mathrm{~A}$ as therapeutic targets in various disease models, Life Sci. 210 (2018) 40-46, https://doi.org/10.1016/j.lfs. 2018.08.063.

[43] P. Cohen, S. Klumpp, D.L. Schelling, An improved procedure for identifying and quantitating protein phosphatases in mammalian tissues, FEBS Lett. 250 (1989) 596-600, https://doi.org/10.1016/0014-5793(89)80803-8.

[44] Y. Xing, Z. Li, Y. Chen, et al., Structural mechanism of demethylation and inactivation of protein phosphatase 2A, Cell 133 (2008) 154-163, https://doi.org/10. 1016/j.cell.2008.02.041.

[45] R. Yabe, A. Miura, T. Usui, et al., Protein phosphatase methyl-esterase PME-1 protects protein phosphatase 2A from Ubiquitin/Proteasome degradation, PLoS One 10 (2015) e0145226, , https://doi.org/10.1371/journal.pone.0145226.

[46] S. LONGIN, J. JORDENS, E. MARTENS, et al., An inactive protein phosphatase 2A population is associated with methylesterase and can be re-activated by the phosphotyrosyl phosphatase activator, Biochem. J. 380 (2004) 111-119, https://doi. org/10.1042/bj20031643.

[47] Y. Xu, Y. Chen, P. Zhang, P.D. Jeffrey, Y. Shi, Structure of a protein phosphatase 2A holoenzyme: insights into B55-Mediated tau dephosphorylation, Mol. Cell 31 (2008) 873-885, https://doi.org/10.1016/j.molcel.2008.08.006.

[48] E. Di Daniel, C.P. Glover, E. Grot, et al., Prolyl oligopeptidase binds to GAP-43 and functions without its peptidase activity, Mol. Cell. Neurosci. 41 (2009) 373-382, https://doi.org/10.1016/j.mcn.2009.03.003.

[49] T. Matsuda, M. Sakaguchi, S. Tanaka, T. Yoshimoto, M. Takaoka, Prolyl oligopeptidase is a glyceraldehyde-3-phosphate dehydrogenase-binding protein that regulates genotoxic stress-induced cell death, Int. J. Biochem. Cell Biol. 45 (2013) 850-857, https://doi.org/10.1016/j.biocel.2013.01.009.

[50] K. Kaszuba, T. Róg, R. Danne, et al., Molecular dynamics, crystallography and mutagenesis studies on the substrate gating mechanism of prolyl oligopeptidase, Biochimie 94 (2012) 1398-1411, https://doi.org/10.1016/j.biochi.2012.03.012.

[51] A. Tsirigotaki, R.V. Elzen, P.V. Veken, A.M. Lambeir, A. Economou, Dynamics and ligand-induced conformational changes in human prolyl oligopeptidase analyzed by hydrogen/deuterium exchange mass spectrometry, Sci. Rep. 7 (2017) 2456 https://doi.org/10.1038/s41598-017-02550-1.

[52] A.M. Slupe, R.A. Merrill, S. Strack, Determinants for substrate specificity of protein phosphatase 2A, Enzyme Res. 2011 (2011) 398751, , https://doi.org/10.4061/ 2011/398751.

[53] P. Seshacharyulu, P. Pandey, K. Datta, S.K. Batra, Phosphatase: PP2A structural importance, regulation and its aberrant expression in cancer, Cancer Lett. 335 (2013) 9-18, https://doi.org/10.1016/j.canlet.2013.02.036.

[54] J.M. Sontag, E. Sontag, Protein phosphatase 2A dysfunction in Alzheimer's disease, Front. Mol. Neurosci. 7 (2014) 16, https://doi.org/10.3389/fnmol.2014.00016.

[55] H.J. Park, K.W. Lee, E.S. Park, et al., Dysregulation of protein phosphatase 2A in parkinson disease and dementia with lewy bodies, Ann. Clin. Transl. Neurol. 3 (2016) 769-780, https://doi.org/10.1002/acn3.337.

[56] C.M. O'Connor, A. Perl, D. Leonard, J. Sangodkar, G. Narla, Therapeutic targeting of 
PP2A, Int. J. Biochem. Cell Biol. (2017), https://doi.org/10.1016/j.biocel.2017.10. 008.

[57] J. Westermarck, Targeted therapies don't work for a reason; the neglected tumor suppressor phosphatase PP2A strikes back, FEBS J. (2018), https://doi.org/10. 1111 /febs.14617 0.

[58] I. Cristobal, L. Garcia-Orti, C. Cirauqui, et al., PP2A impaired activity is a common event in acute myeloid leukemia and its activation by forskolin has a potent antileukemic effect, Leukemia 25 (2011) 606-614, https://doi.org/10.1038/leu.2010. 294.

[59] J. Sangodkar, C.C. Farrington, K. McClinch, et al., All roads lead to PP2A: exploiting the therapeutic potential of this phosphatase, FEBS J. 283 (2016) 1004-1024, https://doi.org/10.1111/febs.13573.

[60] P.T. Männistö, J.A. Garcia-Horsman, Mechanism of action of prolyl oligopeptidase (PREP) in degenerative brain diseases: has peptidase activity only a modulatory role on the interactions of PREP with proteins? Front. Aging Neurosci. 9 (2017) 27, https://doi.org/10.3389/fnagi.2017.00027.

[61] V. Fülop, Z. Bocskei, L. Polgar, Prolyl oligopeptidase: an unusual beta-propeller domain regulates proteolysis, Cell. 94 (1998) 161-170.

[62] A. Kaur, J. Westermarck, Regulation of protein phosphatase 2A (PP2A) tumor suppressor function by PME-1, Biochem. Soc. Trans. 44 (2016) 1683-1693, https:// doi.org/10.1042/bst20160161.

[63] S. Longin, K. Zwaenepoel, E. Martens, et al., Spatial control of protein phosphatase 2A (de)methylation, Exp. Cell Res. 314 (2008) 68-81, https://doi.org/10.1016/j. yexcr.2007.07.030.

[64] S. Tang, C. Lu, L. Mo, et al., Hydrogen peroxide redistributes the localization of protein phosphatase methylesterase 1, Life Sci. 213 (2018) 166-173, https://doi. org/10.1016/j.lfs.2018.10.029.

[65] J.B. Jackson, D.C. Pallas, Circumventing cellular control of PP2A by methylation promotes transformation in an Akt-dependent manner, Neoplasia 14 (2012) 585-599.

[66] R.C. Widau, Y. Jin, S.A. Dixon, B.E. Wadzinski, P.J. Gallagher, Protein phosphatase 2A (PP2A) holoenzymes regulate death-associated protein kinase (DAPK) in ceramide-induced anoikis, J. Biol. Chem. 285 (2010) 13827-13838, https://doi.org/ 10.1074/jbc.M109.085076.

[67] A.M.S.T. Yeasmin, T.M. Waliullah, A. Kondo, et al., Orchestrated action of PP2A antagonizes Atg13 phosphorylation and promotes autophagy after the inactivation of TORC1, PLoS One 11 (2016) e0166636, , https://doi.org/10.1371/journal.pone. 0166636.

[68] P.-M. Wong, Y. Feng, J. Wang, R. Shi, X. Jiang, Regulation of autophagy by coordinated action of mTORC1 and protein phosphatase 2A, Nat. Commun. 6 (2015) 8048, https://doi.org/10.1038/ncomms9048.

[69] V. Cianfanelli, C. Fuoco, M. Lorente, et al., AMBRA1 links autophagy to cell proliferation and tumorigenesis by promoting c-Myc dephosphorylation and degradation, Nat. Cell Biol. 17 (2014) 20, https://doi.org/10.1038/ncb3072.

[70] G. Shohat, T. Spivak-Kroizman, O. Cohen, et al., The pro-apoptotic function of death-associated protein kinase is controlled by a unique inhibitory autophosphorylation-based mechanism, J. Biol. Chem. 276 (2001) 47460-47467, https:// doi.org/10.1074/jbc.M105133200.

[71] V. Levin-Salomon, S. Bialik, A.J.A. Kimchi, DAP-kinase and autophagy, Apoptosis 19 (2014) 346-356, https://doi.org/10.1007/s10495-013-0918-3.

[72] K.A. Puttonen, S. Lehtonen, A. Raasmaja, P.T. Männistö, A prolyl oligopeptidase inhibitor, Z-Pro-Prolinal, inhibits glyceraldehyde-3-phosphate dehydrogenase translocation and production of reactive oxygen species in CV1-P cells exposed to 6hydroxydopamine, Toxicol. Vitr. 20 (2006) 1446-1454, https://doi.org/10.1016/j. tiv.2006.07.001.

[73] C. He, M.C. Bassik, V. Moresi, et al., Exercise-induced BCL2-regulated autophagy is required for muscle glucose homeostasis, Nature 481 (2012) 511, https://doi.org/ 10.1038/nature10758.

[74] X. Sui, N. Kong, L. Ye, et al., p38 and JNK MAPK pathways control the balance of apoptosis and autophagy in response to chemotherapeutic agents, Cancer Lett. 344
(2014) 174-179.

[75] M.J. Moreno-Baylach, K.A. Puttonen, J. Tenorio-Laranga, et al., Prolyl endopeptidase is involved in cellular signalling in human neuroblastoma SH-SY5Y cells, Neurosignals 19 (2011) 97-109, https://doi.org/10.1159/000326342.

[76] S. Tanaka, K. Suzuki, M. Sakaguchi, The prolyl oligopeptidase inhibitor SUAM14746 attenuates the proliferation of human breast cancer cell lines in vitro, Breast Cancer 24 (2017) 658-666, https://doi.org/10.1007/s12282-017-0752-5.

[77] G. Larrinaga, I. Perez, L. Blanco, et al., Prolyl endopeptidase activity is correlated with colorectal cancer prognosis, Int. J. Med. Sci. 11 (2014) 199-208, https://doi. org/10.7150/ijms.7178.

[78] J. Tenorio-Laranga, F. Coret-Ferrer, B. Casanova-Estruch, M. Burgal, J.A. GarcíaHorsman, Prolyl oligopeptidase is inhibited in relapsing-remitting multiple sclerosis, J. Neuroinflammation 7 (2010) 23, https://doi.org/10.1186/1742-20947-23.

[79] J. Tenorio-Laranga, C. Montoliu, A. Urios, et al., The expression levels of prolyl oligopeptidase responds not only to neuroinflammation but also to systemic inflammation upon liver failure in rat models and cirrhotic patients, $\mathrm{J}$. Neuroinflammation 12 (2015) 183, https://doi.org/10.1186/s12974-015-0404-7.

[80] T.A. Natunen, M. Gynther, H. Rostalski, K. Jaako, A.J. Jalkanen, Extracellular prolyl oligopeptidase derived from activated microglia is a potential neuroprotection target, Basic Clin. Pharmacol. Toxicol. 124 (2019) 40-49, https://doi.org/10.1111/ bcpt.13094.

[81] J. Tenorio-Laranga, C. Montoliu, A. Urios, et al., The expression levels of prolyl oligopeptidase responds not only to neuroinflammation but also to systemic inflammation upon liver failure in rat models and cirrhotic patients, J. Neuroinflammation 12 (2015) 183, https://doi.org/10.1186/s12974-015-0404-7.

[82] M.J. Hannula, T.T. Myöhänen, J. Tenorio-Laranga, P.T. Männistö, J.A. GarciaHorsman, Prolyl oligopeptidase colocalizes with alpha-synuclein, beta-amyloid, Tau Protein and Astroglia in the post-mortem brain samples with Parkinson's and Alzheimer's diseases, Neuroscience 242 (2013) 140-150, https://doi.org/10.1016/ j.neuroscience.2013.03.049.

[83] M. Sakaguchi, T. Matsuda, E. Matsumura, T. Yoshimoto, M. Takaoka, Prolyl oligopeptidase participates in cell cycle progression in a human neuroblastoma cell line, Biochem. Biophys. Res. Commun. 409 (2011) 693-698, https://doi.org/10. 1016/j.bbrc.2011.05.066.

[84] N. Wlodarchak, Y. Xing, PP2A as a master regulator of the cell cycle, Crit. Rev. Biochem. Mol. Biol. 51 (2016) 162-184, https://doi.org/10.3109/10409238.2016. 1143913.

[85] J.D. Monroe, R.D. Heathcote, Protein phosphatases regulate the growth of developing neurites, Int. J. Dev. Neurosci. 31 (2013) 250-257, https://doi.org/10.1016/ j.ijdevneu.2013.01.005.

[86] K. Sabapathy, Role of the JNK pathway in human diseases, in: S. Shenolikar (Ed.), Progress in Molecular Biology and Translational Science, Academic Press, 2012, pp. $145-169$.

[87] R. Liu, X.W. Zhou, H. Tanila, et al., Phosphorylated PP2A (tyrosine 307) is associated with Alzheimer neurofibrillary pathology, J. Cell. Mol. Med. 12 (2008) 241-257, https://doi.org/10.1111/j.1582-4934.2008.00249.x.

[88] D. Mondaca-Ruff, J.A. Riquelme, C. Quiroga, et al., Angiotensin II-Regulated autophagy is required for vascular smooth muscle cell hypertrophy, Front. Pharmacol. 9 (2019), https://doi.org/10.3389/fphar.2018.01553.

[89] J. Willemsen, O. Wicht, J.C. Wolanski, et al., Phosphorylation-dependent feedback inhibition of RIG-I by DAPK1 identified by kinome-wide siRNA screening, Mol. Cell 65 (2017) 403-415, https://doi.org/10.1016/j.molcel.2016.12.021 e408.

[90] K. Sikorski, A. Mehta, M. Inngjerdingen, et al., A high-throughput pipeline for validation of antibodies, Nat. Methods 15 (2018) 909-912, https://doi.org/10.1038/ S41592-018-0179-8.

[91] J.D. Kim, C. Toda, G. D'Agostino, et al., Hypothalamic prolyl endopeptidase (PREP) regulates pancreatic insulin and glucagon secretion in mice, Proc. Natl. Acad. Sci. U. S. A. 111 (2014) 11876-11881, https://doi.org/10.1073/pnas.1406000111. 